\title{
Etiology of Respiratory Diseases of Poultry Farms in the North Coast of Egypt
}

\author{
Hanan Saad El-Samahy and Disouky Mohamed Mourad ${ }^{*}$ \\ Department of Animal and Poultry Health, Division of Animal and Poultry Production, Desert Research Center, Ministry of Agriculture, 1-Mathaf El- \\ Materia Street, Cairo, 2633759, Egypt \\ *Corresponding author’s Email: dismou235@gmail.com; ORCID: 0000-0001-7777-9305
}

Received: 08 Jan. 2021

Accepted: 25 Feb. 2021

\begin{abstract}
The current study aimed to identify the respiratory problems in poultry farms located in the north coast of Egypt from October 2018 to November 2019. A total of 89 poultry flocks (79 broilers, 5 layers, 3 ducks, and 2 turkeys) were investigated for four major viral respiratory pathogens, namely avian influenza (AI) H9N2, AI H5 subtypes, Newcastle Disease (ND), and Infectious Bronchitis (IB) viruses. All 89 flocks were subjected to real-time PCR to investigate AI H9N2 virus. The samples of 31, 43, and 15 out of 89 flocks were selected for the investigation of ND, IB, and AI H5 subtypes viruses, respectively, using real-time PCR. Sample selection was performed according to the mortalities, clinical signs, and post mortem lesions. The positive findings indicated that 22 out of 89 flocks were positive for AI H9N2 virus (2 layers +20 broilers), 32 out of 43 flocks were positive for IB virus (2 layers +30 broilers), 24 out of 31 flocks were positive for ND virus (1 Duck +1 layer+ 22 broilers) and 9 out of 15 flocks were positive for AI H5N8 virus (1 turkey +1 duck +7 broilers). Partial sequencing for selected isolates of six ND, five IB, four H9N2, and three H5N8 viruses was applied, then nucleotide sequences were accessed on GenBank. Six ND isolates belonged to genotype Vll viruses circulating in Egypt. Two IB isolates were related to the classical strain circulating in Egypt, while the other three IB isolates belonged to EGY/Variant 11. Four H9N2 AI isolates were related to G1-lineage of H9 viruses circulating in the Middle East and Egypt. Three H5N8 AI isolates belonged to the highly diverse clade 2.3.4.4.b viruses circulating in Egypt. It was concluded that ND and IB viruses isolated in this study were not related to their vaccinal strains. Also, AI H5N8 circulating alone in affected flocks while AI H9N2 circulating alone and/or mixed with either IB or ND viruses. Finally, there is a need to devise a complete strategy to control the isolated respiratory viruses on the north coast of Egypt.
\end{abstract}

Keywords: Poultry, Respiratory, RRT-PCR, Sequence, Viruses

\section{INTRODUCTION}

Respiratory affection is a major problem in the commercial poultry industry leading to the annual loss of a hundred million dollars (Easterday et al.,1997). During the last few years, Egyptian commercial chicken flocks have been suffering from the co-circulation of multiple respiratory viruses leading to variable mortality rates and different clinical manifestations. Many important diseases can affect the respiratory system of poultry, including Avian Influenza (AI), Newcastle Disease (ND), and Infectious Bronchitis (IB) viruses (Shankar, 2008). The AI-H5N1 viruses affected poultry flocks in many countries including Egypt, and have become endemic (Aly et al., 2008). An additional challenge facing the poultry industry occurred in Egypt when AI-H9N2 subtype was detected during 2010-11 in chickens and commercial quails (El-
Zoghby et al., 2012). In addition to high pathogenic avian influenza (HPAI) $\mathrm{H} 5 \mathrm{~N} 1$ and low pathogenic avian influenza (LPAI) H9N2, there was the incursion of HPAI H5N8 to Egypt in November 2016 via wild birds followed by spreading into commercial poultry flocks, further complicated the situation (Hassan et al., 2019). The ND virus still represents a serious problem for poultry production in many countries, although strict vaccination and other controlling regimes. In Egypt, ND virus strains of recent outbreaks in poultry farms have belonged to class II, genotype VII which might be introduced through the trading of poultry and poultry products with China and the Middle Eastern countries (Mohamed et al., 2011; Radwan et al., 2013). Furthermore, IB virus threatens the poultry industry worldwide and is considered as one of the most economically important respiratory viral diseases (Cook et al., 2012), and it was described as a natural infection in 
different countries of Asia and the Middle-East in association with both HPAI and/or LPAI virus (Hassan et al., 2016). Therefore, the present study aimed to determine the current field situation of these major avian respiratory pathogens in the north coast region with the molecular identification of certain selected isolates to monitor and record their genetic properties through sequencing, phylogenetic analysis, and GenBank accessions.

\section{MATERIALS AND METHODS}

\section{Samples collection and processing}

Tracheal and/or oropharyngeal swabs and tissues of 89 different poultry flocks (10 samples per each flock) suffering from respiratory symptoms, a severe drop in egg production, and high mortalities were collected between October 2018 and December 2019. The area of investigation included the north coast area (west Alexandria, El-Hamam, El Alamein, and Matrouh governorates) in Egypt. All 89 flocks were subjected to real-time PCR for the investigation of AI H9N2 virus. The samples of 31,43 , and 15 out of 89 flocks were selected for the investigation of ND, IB, and AI H5 subtypes viruses, respectively, using real-time PCR. Sample selection was performed according to the mortalities, clinical signs, and post mortem lesions. Tissue samples were grounded in phosphate buffer saline with a $\mathrm{pH}$ of 7.0 to 7.4 containing gentamycin $(50 \mu \mathrm{g} / \mathrm{mL})$ and Mycostatin $(1,000$ units $/ \mathrm{mL})$ in a 1:5 (w/v) dilution, centrifuged, and tissue supernatant was collected (OIE, 2005; Naguib et al., 2017). The transport medium used for collected swabs composed of glycerol (50\%), Phosphate buffer saline (PBS) $(50 \%)$ with $2 \times 10^{6} \mathrm{U} /$ liter penicillin, $200 \mathrm{mg} /$ liter streptomycin, and $250 \mathrm{mg} / \mathrm{liter}$ amphotericin B. Samples were stored at $-80^{\circ} \mathrm{C}$ until being tested (Gelb and Jackwood, 2008). All tissue samples were tested using RRT-PCR, then certain selected positive samples for each virus were purified through intra-allantoic inoculation of Specific Pathogen Free Embryonated Chicken Egg (SPFECE, De Wit, 2000), and these allantoic fluids were subjected to RT-PCR and sequencing.

\section{Viral RNA extraction}

All steps were carried out according to the manufacturer's instructions. Whole nucleic acid extraction from the samples was performed using the QIAamp® minielute virus spin kit (Qiagen, Germany, GmbH). Briefly, $200 \mu \mathrm{l}$ of the sample suspension was incubated with $25 \mu \mathrm{l}$ of Qiagen protease, and $200 \mu \mathrm{l}$ of $\mathrm{AL}$ lysis buffer at $56^{\circ} \mathrm{C}$ for $15 \mathrm{~min}$, then $250 \mu \mathrm{l}$ of ethanol $100 \%$ was added to the lysate. After that, the sample was washed and centrifuged. The nucleic acid was eluted with $100 \mu \mathrm{l}$ of elution buffer.

\section{Real-time reverse transcription-polymerase chain reaction}

For AI viruses, type A (matrix) gene primer was used (Spackman et al., 2002), followed by H5 (Löndt et al., 2008), H9 (Ben Shabat et al., 2010), and N8 primers (Hoffmann et al., 2016), while for ND virus and IB virus, $\mathrm{M}$ primer and $\mathrm{N}$ primer were utilized, respectively (Wise et al., 2004; Meir et al., 2010). Viral RNA extraction with QIAamp® was performed using viral RNA mini kit buffers (Qiagen, Germany) and Master Mix kit (Quantitect probe RT_PCR kit), and all steps were carried out according to the manufacturer's instructions (Table 1).

\section{Conventional polymerase chain reaction}

The $\mathrm{H}$ gene primer was used with the length of amplified product $311 \mathrm{bp}$ and $920 \mathrm{bp}$ for AI H5 virus and AI H9 virus, respectively (Slomka et al., 2007; Adel et al., 2016), while for ND virus, M \& F gene primer with the length of amplified product 766 bp (Mase et al., 2002), and for IB virus, Spike SP1 gene primer with the length of amplified product 457 bp were used (Naguib et al., 2017). Viral RNA extraction with QIAamp® was performed using viral RNA mini kit buffers (Qiagen, Germany) and Master Mix kit (Quantitect probe RT_PCR kit), and all steps were performed according to the manufacturer's instructions (Table 2).

\section{Analysis of the polymerase chain reaction products \\ The products of PCR were separated by} electrophoresis on agarose gel $1.5 \%$ (Applichem, Germany, GmbH) in 1x Tris Borate EDTA (TBE) buffer at room temperature using gradients of $5 \mathrm{~V} / \mathrm{cm}$. For gel analysis, $15 \mu$ of the products were loaded in each gel slot. A gene ruler 100 bp DNA ladder (Fermentas, Thermofisher, Germany) was used to determine the fragment sizes. The gel was photographed by a gel documentation system (Alpha Innotech, Biometra), and the data were analyzed using the computer software (Automatic Image Capture Software, protein simple formerly cell biosciences, USA). 


\section{Sequencing and phylogenetic analysis}

The PCR products of selected different isolated respiratory viruses (six ND, five $\mathrm{IB}$, four $\mathrm{H} 9$, and three $\mathrm{H} 5$ subtypes viruses) were purified using QIAquick PCR Product extraction kit (Qiagen, Valencia). Bigdye Terminator V3.1 cycle sequencing kit (Perkin-Elmer) was used for the sequence reaction, and then it was purified using Centrisep spin column. Sequence analysis was done by Applied Biosystems3130 genetic analyzer (HITACHI, Japan), and the sequence identity was initially performed by A BLAST ${ }^{\circledR}$ analysis (Basic Local Alignment Search Tool) (Altschul et al., 1990) to be established to GenBank accessions. The phylogenetic tree was created by the MegAlign module of Laser gene DNAStar version 12.1 (Thompson et al., 1994), and Phylogenetic analyses were performed using maximum likelihood, neighbor-joining, and maximum parsimony in MEGA6 (Tamura et al., 2013).

\section{Ethical approval}

The present study was affirmed by the Ethics of Animal Health Committee, Desert Research Center, Egypt.

Table 1. Oligonucleotide primers and probes used for RRT-PCR.

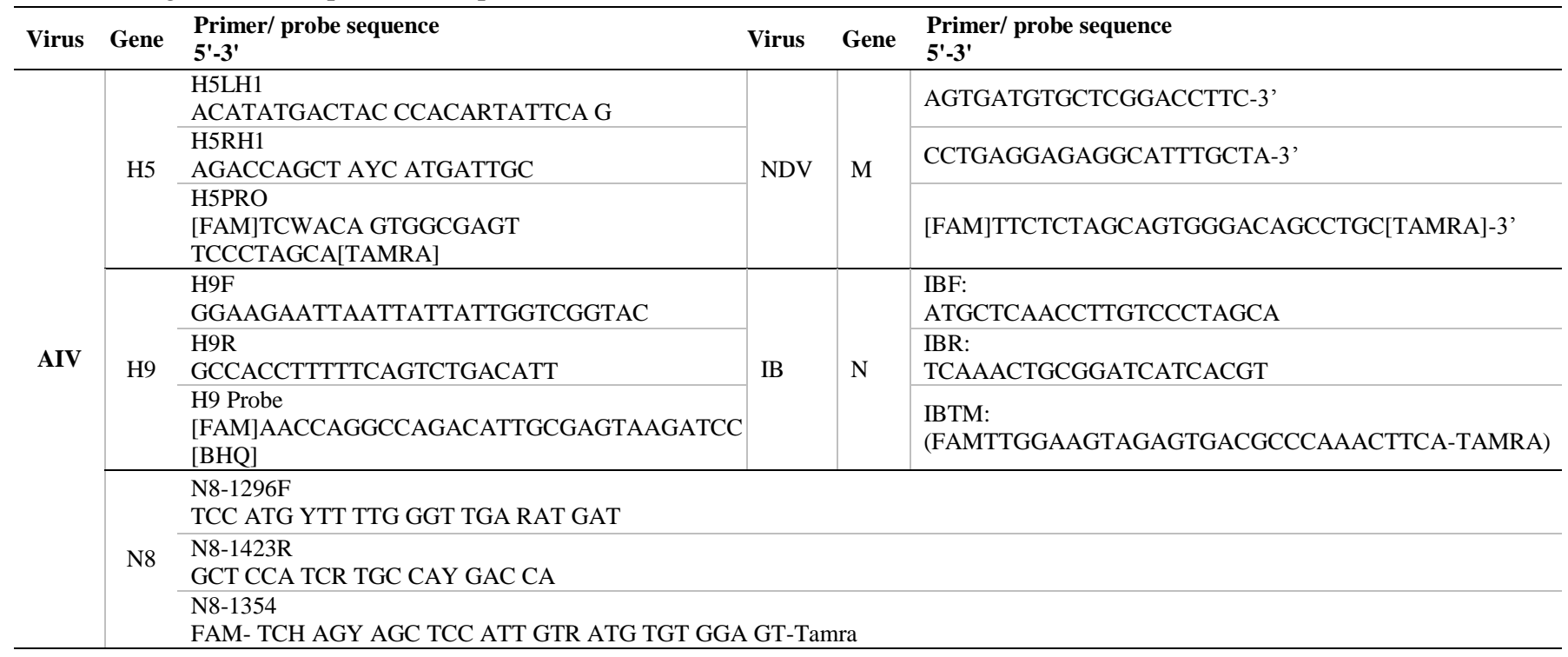

Table 2. Oligonucleotide primers used for conventional PCR.

\begin{tabular}{|c|c|c|c|}
\hline Target agent & Target gene & $\begin{array}{l}\text { Primer sequence } \\
\left(5^{\prime}-3^{\prime}\right)\end{array}$ & $\begin{array}{l}\text { Length of amplified } \\
\text { product (bp) }\end{array}$ \\
\hline \multirow{2}{*}{ H9 } & \multirow{2}{*}{$\mathrm{H}$} & $\begin{array}{l}\text { H9F } \\
\text { GGAAGAATTAATTATTATTGGTCGGTAC }\end{array}$ & \multirow{2}{*}{920 bp } \\
\hline & & $\begin{array}{l}\text { HT7R } \\
\text { TAA TAC GAC TCA CTA TAA GTA CAA ACA AGG GTG }\end{array}$ & \\
\hline \multirow{3}{*}{ IB } & \multirow{3}{*}{ Spike SP1 } & IBV-HVR1-2-FW & \multirow{3}{*}{457 bp } \\
\hline & & GTK TAC TACTAC CAR AGT GC & \\
\hline & & $\begin{array}{l}\text { IBV-HVR1-2-RV } \\
\text { GAA GTG RAA ACR AGA TCA CCA TTT A }\end{array}$ & \\
\hline \multirow{2}{*}{ ND } & \multirow{2}{*}{$\mathrm{M}$ and $\mathrm{F}$} & $\begin{array}{l}\text { M2 } \\
\text { TGG-AGC-CAA-ACC-CGC-ACC-TGC-GG }\end{array}$ & \multirow{2}{*}{$766 \mathrm{bp}$} \\
\hline & & $\begin{array}{l}\text { F2 } \\
\text { GGA-GGA-TGT-TGG-CAG-CAT-T }\end{array}$ & \\
\hline \multirow{2}{*}{ H5 } & \multirow{2}{*}{$\mathrm{H}$} & $\begin{array}{l}\text { H5-kha-1 } \\
\text { CCT CCA GAR TAT GCM TAY AAA ATT GTC }\end{array}$ & \multirow{2}{*}{$311 \mathrm{bp}$} \\
\hline & & $\begin{array}{l}\text { H5-kha-3 } \\
\text { TAC CAA CCG TCT ACC ATK CCY TG }\end{array}$ & \\
\hline
\end{tabular}




\section{RESULTS}

\section{Prevalence of respiratory viruses}

Concerning the results of the RRT-PCR used for detection of respiratory viruses (Figure 1), 22 out of 89 flocks $(24.7 \%)$ were positive (+ve) for AI H9N2 virus, 9 out of 15 flocks $(60 \%)$ were positive for AI H5 subtype virus, 24 out of 31 flocks $(77.4 \%)$ were positive for ND virus, and 32 out of 43 flocks (74.4\%) were positive for IB virus (Tables 3 and 4). All AI H5 subtypes were H5N8 viruses.

\section{Single and mixed viral infection}

There were 16 out of 89 poultry flocks negative for RRT-PCR. Furthermore, 59 flocks were recorded with a single viral infection, 8 flocks with AI H9N2, 22 with IB, 20 with ND, and 9 with AI H5N8 viruses. Mixed infection with AI H9N2 virus occurred in 14 poultry flocks, 10 flocks with mixed IB, and 4 flocks with mixed ND virus. On the other hand, there was no mixed infection with AI H5N8 subtype virus (Table 5).

Results of conventional polymerase chain reaction

The PCR product revealed the specific amplification of 920, 766, 457, and $311 \mathrm{bp}$ fragments for all selected AI $\mathrm{H} 9 \mathrm{~N} 2$, ND, IB, and AI H5N8 isolates of viruses, respectively (Figure 2 ).

Sequencing and phylogenetic analysis of avian influenza H9N2 isolated viruses

The isolates of four selected AI H9N2 viruses were genetically related to G1-lineage of $\mathrm{H} 9$ viruses circulating in the Middle East and were clustered in the same branch of the isolated Egyptian viruses during 2011-2019. The phylogenetic tree of HA gene showed that the first three analyzed isolates (A/Chicken/Egypt/North-coast/19 H9N2, A/Chicken/Egypt/Bahig/19 H9N2, and A/Chicken/Egypt/Matrouh/19 H9N2) were genetically related and significantly closer to each other (99 bootstrap value), and with the fourth isolate (A/chicken/Egypt/KingMariot/18 H9N2) 54 bootstrap value (Figure 3). KingMariot 2018 AI H9N2 virus isolate had an identity of $98.7 \%$ with other selected isolates which were closely related $(100 \%)$ to each other. The four selected isolates were identical with other Egyptian strains of 2011-2019 by 94.8-100\%, while with Hong Kong strains in 1997 were identical by $87.3-87.4 \%$, Israel strains isolated in 2018 were identical with the four isolates from $97.1-97.2 \%$ and Korean strains in 1996 and 2007 had the identity of 82.7$83.1 \%$ (Figure 4).

Sequencing and phylogenetic analysis of avian influenza $\mathrm{H5N8}$ isolated viruses

The selected three H5N8 HPAIV isolates were compared with Egyptian strains during 2014-2019, and it was found that these isolates belonged to the highly diverse clade 2.3.4.4.b viruses circulating in Egypt. On the basis of the phylogenetic tree, the analyzed isolates
(A/chicken/Egypt/El-hamam/1/2019

$\mathrm{H} 5 \mathrm{~N} 8$,

A/chicken/Egypt/El-hamam/10/2019 H5N1, and A/turkey/Egypt/North-coast k38/2019 H5N1) were clustered together and related highly genetically of 86 bootstrap value with each other, and 53 bootstrap value with other Egyptian viruses isolated during 2014-2019 (Figure 5). An amino acid sequence identity of the three isolates was $100 \%$ with each other and $98.9-99.3 \%$ with the Egyptian strains from 2017 to 2019 (Figure 6).

\section{Sequencing and phylogenetic analysis of} infectious bronchitis isolated viruses

The isolates of five selected IB viruses were compared with Egyptian classic, variant, QX, and their vaccinal strains. Two IB isolates (MT324521_IBV Matrouh and MT324522_IBV El-hamam) were genetically related to the classical strain circulating in Egypt (71 bootstrap value), and 33 bootstrap value with each other. The mentioned isolates had an amino acid sequence identity of $100 \%$ with each other and 71.8 91.2\% with the Egyptian strains. The other three IB isolates (MT324523_IBV North-coast-k70, MT324524_IBV El-Alamin, and MT324525_IBV Elhawaria) belonged to EGY/Variant 11. MT324523_IBV North-coast-k70, and MT324525_IBV El-hawaria were genetically related to each other, 97 bootstrap value with an amino acid sequence identity of $100 \%$, and had 41 bootstrap value with an amino acid sequence identity of $97.5 \%$ and 70.6-75.2\% when compared with MT324524_IBV El-Alamin and the Egyptian strains respectively (Figures 7 and 8).

Sequencing and phylogenetic analysis of Newcastle disease viruses

The isolates of all selected six ND viruses were compared with Egyptian strains of 2012- 2018. The findings indicated that the isolates belonged to the genotype Vll viruses circulating in Egypt. The phylogenetic tree revealed that the analyzed isolates, MT324529_North coast-4 and MT324530_North coast-5 were closely related to each other, 66 bootstrap values. MT324531_North coast-6 virus isolate was clustered separately alone, 63 bootstrap value, while the other three virus isolates (MT324528_North coast-3, MT324526_North coast-1, and MT324527_North coast-2) were clustered together, and related genetically to each other with 57 bootstrap value (Figure 9). All selected six isolates showed an amino acid sequence identity of 98.6$100 \%$ with each other, and $97.6-100 \%$ and $97.6-98.1 \%$ when compared with the Egyptian and Israel strains respectively (Figure 10).

\section{isolates}

GenBank accessions of all selected viruses'

Nucleotides sequences of all selected AI H9N2, AI H5N8, IB, and ND viruses' isolates were submitted to GenBank, and given accession numbers (Table 6). 


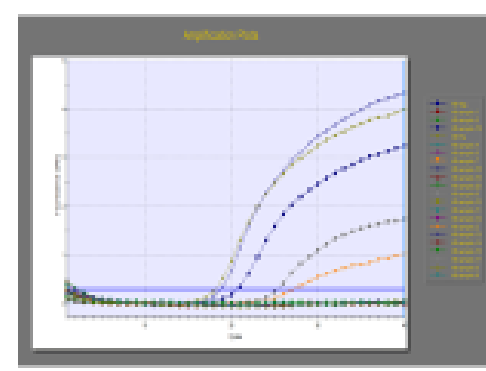

H9 Amplification Plots

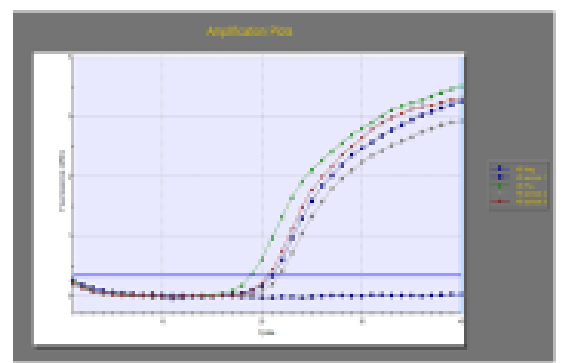

H5 Amplification Plots

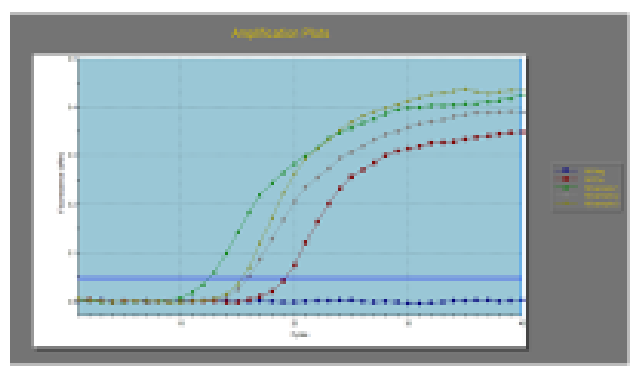

N8 Amplification Plots

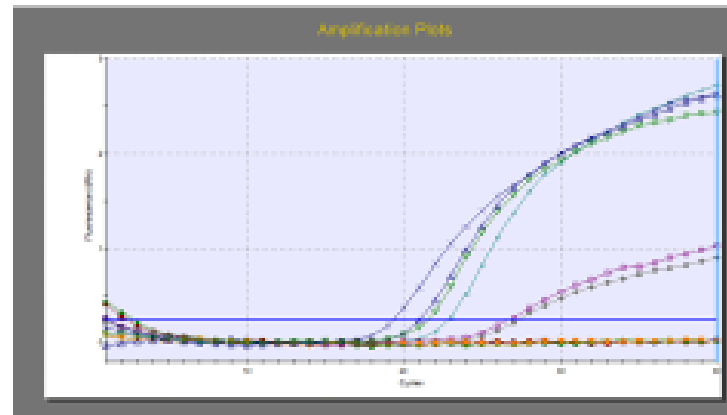

IB Amplification Plots

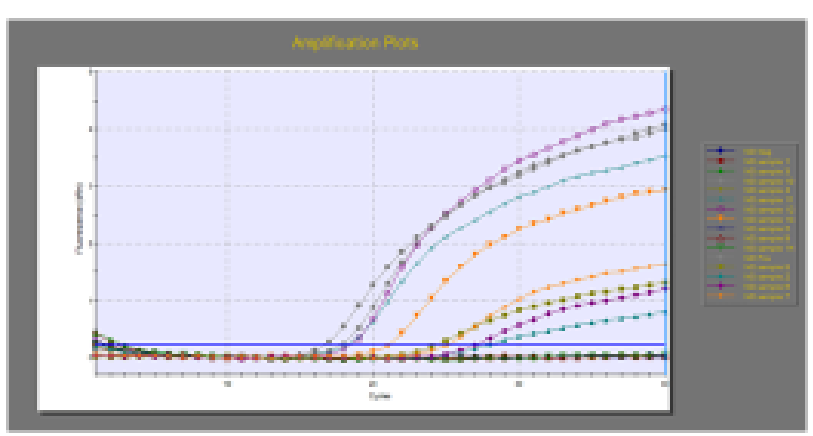

ND Amplification Plots

Figure 1. Results of RRT-PCR for collected samples of different poultry flocks showed amplification plots for the following primers $\mathrm{H} 9, \mathrm{H} 5, \mathrm{~N} 8, \mathrm{M}$, and $\mathrm{N}$ of $\mathrm{H} 9 \mathrm{~N} 2, \mathrm{H} 5 \mathrm{~N} 8, \mathrm{ND}$, and IB viruses' isolates, respectively. ND: Newcastle disease IB: Infectious Bronchitis.

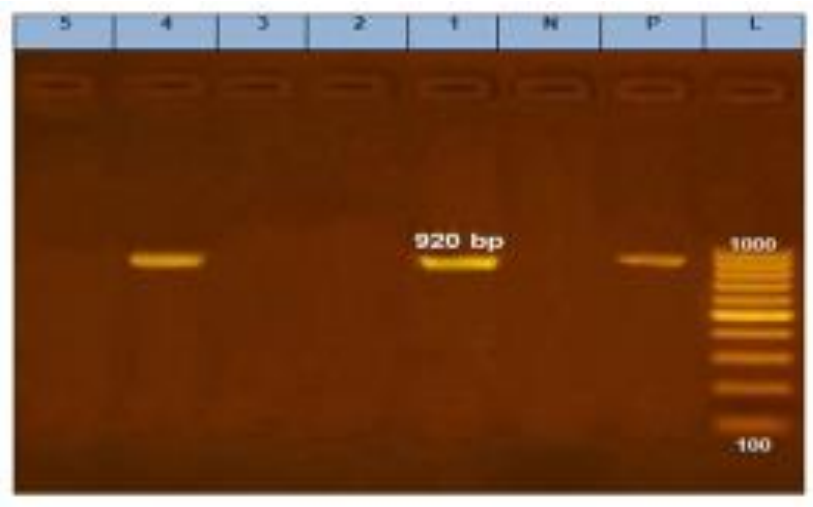

Al $\mathrm{H} 9$ collective photo details

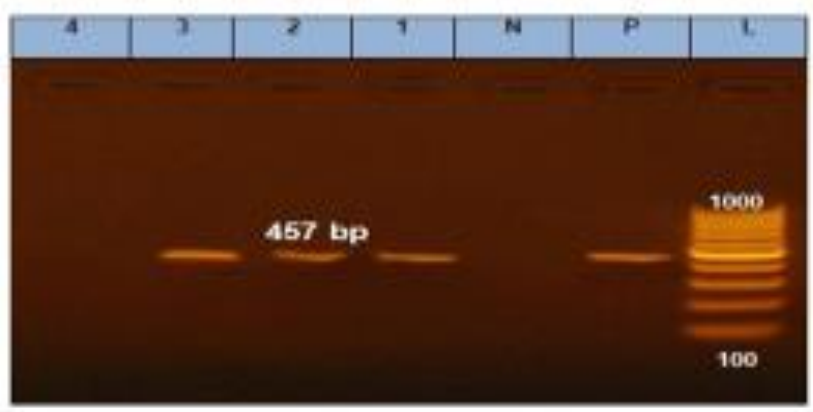

IB collective photo details

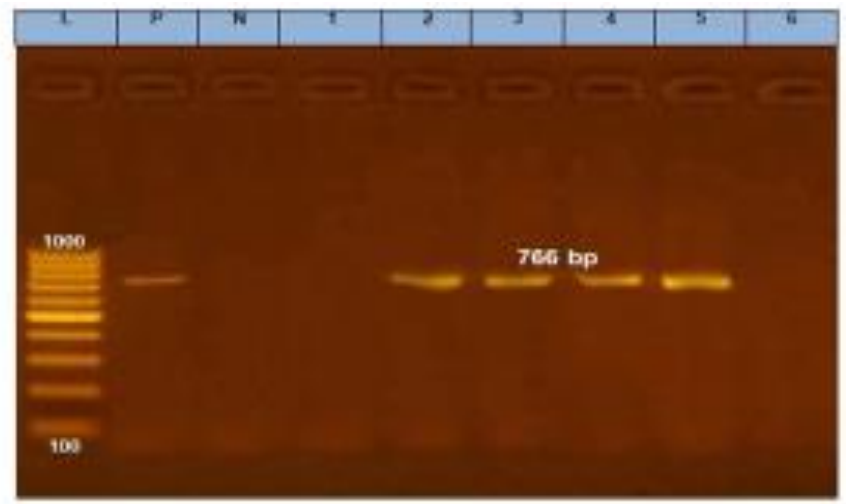

ND collective photo details

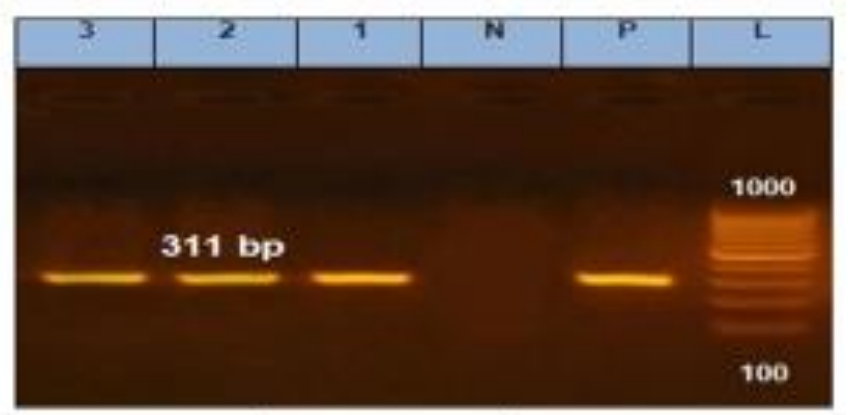

Al H5 collective photo details

Figure 2. Results of conventional PCR showed the specific amplification of 920, 766, 457, and 311 bp fragment for all selected AI H9N2, ND, IB, and AI H5N8 viruses' isolates, respectively. ND: Newcastle disease IB: Infectious Bronchitis. 


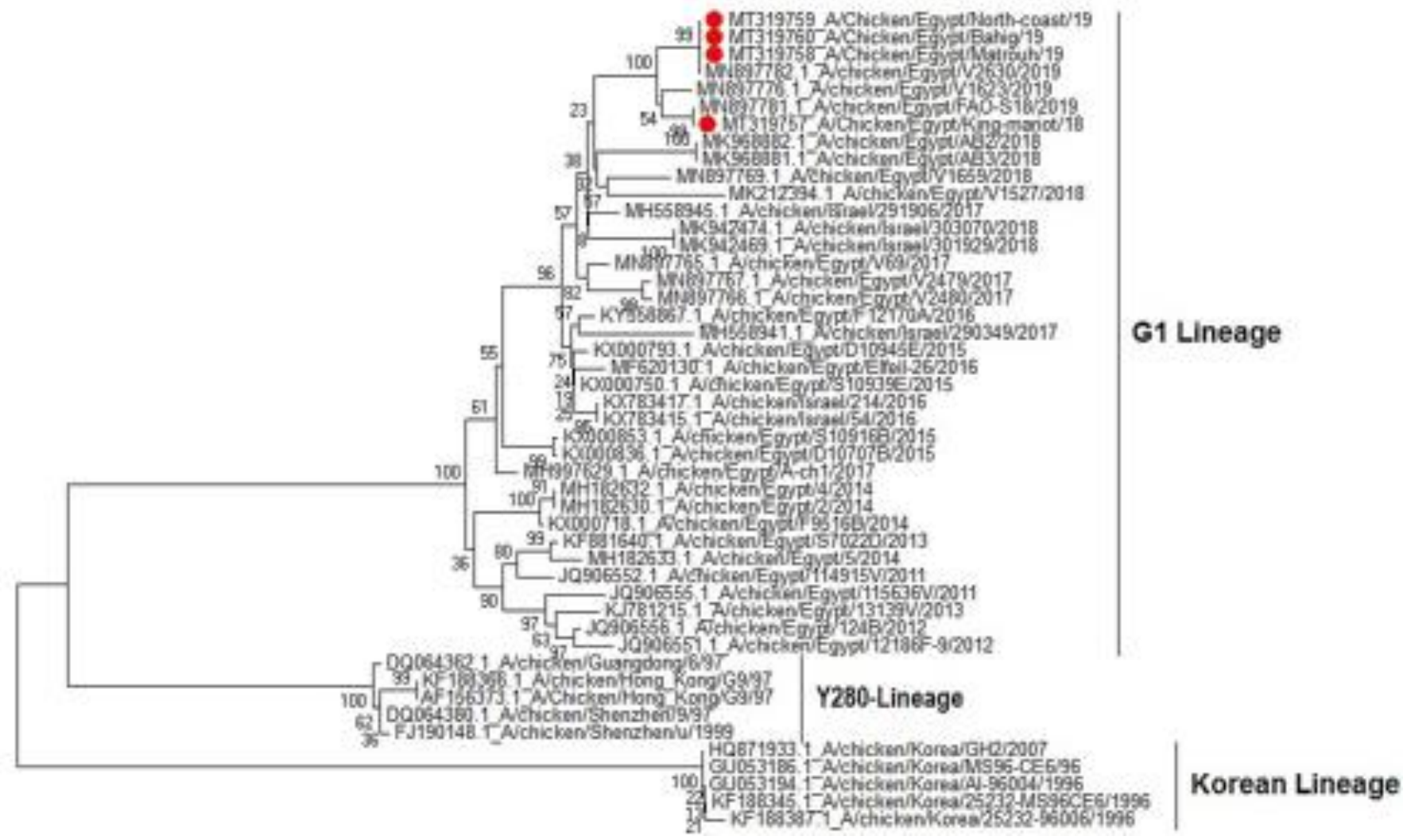

Figure 3. Phylogenetic tree showing the genetic relationships between circulating LPAI H9N2 and the four selected isolates for the HA gene (indicated by red dots).

PercentIdentity

\begin{tabular}{|c|c|c|c|c|c|c|c|c|c|c|c|c|c|c|c|c|c|c|c|c|c|c|c|c|c|c|c|}
\hline & 1 & 2 & 3 & 4 & 5 & 6 & 7 & 8 & 9 & 10 & 11 & 12 & 13 & 14 & 15 & 16 & 17 & 18 & 19 & 20 & 21 & 22 & 23 & 24 & 25 & 26 & \\
\hline 1 & & 8.4 & 98.8 & 97.8 & 98.5 & 97.9 & 97.9 & 97.2 & 97.2 & 96.8 & 98.6 & 96.7 & 95.7 & 95.5 & 95.4 & 94.6 & 95.5 & 95.7 & 83.7 & 83.7 & 88.1 & 88.1 & 95.5 & 95.7 & $95.7 \mathrm{~g}$ & 95.7 & \\
\hline 2 & 1.7 & & 97.9 & 98.9 & 97.5 & 97.4 & 97.4 & 96.7 & 96.7 & 96.4 & 98.1 & 96.2 & 95.2 & 95.4 & 95.2 & 93.9 & 95.1 & 95.2 & 83.6 & 83.6 & 88.5 & 88.5 & 95.1 & 95.2 & 95.29 & 95.2 & 2 \\
\hline 3 & 1.2 & 2.1 & & 97.4 & 99.4 & 97.7 & 97.8 & 96.9 & 96.9 & 96.6 & 98.4 & 96.7 & 95.7 & 95.5 & 95.7 & 94.4 & 95.5 & 95.7 & 84.0 & 84.0 & 87.9 & 87.9 & 95.5 & 95.7 & $95.7 \mathrm{~g}$ & 95.7 & 3 \\
\hline 4 & 2.3 & 1.1 & 2.6 & & 97.1 & 97.2 & 97.3 & 96.5 & 96.5 & 96.1 & 97.8 & 96.1 & 95.1 & 95.3 & 94.7 & 93.8 & 95.0 & 94.8 & 83.6 & 83.6 & 87.9 & 87.9 & 95.0 & 94.8 & $94.8 \mathrm{~g}$ & 94.8 & 4 \\
\hline 5 & 1.5 & 2.5 & 0.6 & 3.0 & & 97.3 & 97.4 & 96.6 & 96.6 & 96.2 & 98.0 & 96.4 & 95.3 & 95.2 & 95.3 & 94.0 & 95.2 & 95.3 & 83.9 & 83.9 & 87.8 & 87.8 & 95.2 & 95.3 & $95.3 \mathrm{~g}$ & 95.3 & 5 \\
\hline 6 & 2.1 & 2.6 & 2.4 & 2.9 & 2.8 & & 97.9 & 97.4 & 97.4 & 97.1 & 98.6 & 96.9 & 95.9 & 95.8 & 95.4 & 94.6 & 95.8 & 95.7 & 83.9 & 83.9 & 88.1 & 88.1 & 95.8 & 95.7 & $95.7 \mathrm{~g}$ & 95.7 & 6 \\
\hline 7 & 2.1 & 2.6 & 2.3 & 2.8 & 2.6 & 2.1 & & 98.0 & 98.0 & 97.7 & 98.8 & 97.9 & 96.8 & 96.6 & 96.6 & 95.5 & 96.7 & 96.6 & 83.2 & 83.2 & 87.8 & 87.8 & 96.7 & 96.6 & $96.6 \mathrm{~g}$ & 96.6 & 7 \\
\hline 8 & 2.9 & 3.4 & 3.1 & 3.6 & 3.5 & 2.6 & 2.0 & & 99.8 & 99.4 & 98.4 & 98.8 & 98.2 & 97.8 & 97.5 & 96.9 & 97.7 & 97.5 & 83.2 & 83.2 & 87.7 & 87.7 & 97.7 & 97.5 & 97.59 & 97.5 & 8 \\
\hline 9 & 2.9 & 3.4 & 3.1 & 3.6 & 3.5 & 2.6 & 2.0 & 0.2 & & 99.6 & 98.6 & 99.1 & 98.5 & 98.0 & 97.8 & 97.2 & 97.7 & 97.5 & 83.2 & 83.2 & 87.7 & 87.7 & 97.7 & 97.5 & 97.59 & 97.5 & 9 \\
\hline 10 & 3.3 & 3.8 & 3.5 & 4.0 & 3.9 & 3.0 & 2.4 & 0.6 & 0.4 & & 98.2 & 98.7 & 98.4 & 97.7 & 97.4 & 96.8 & 97.3 & 97.2 & 83.2 & 83.2 & 87.3 & 87.3 & 97.3 & 97.2 & $97.2 \mathrm{~g}$ & 97.2 & 10 \\
\hline 11 & 1.4 & 1.9 & 1.7 & 2.3 & 2.0 & 1.4 & 1.2 & 1.7 & 1.4 & 1.8 & & 97.9 & 97.1 & 96.8 & 96.8 & 95.8 & 96.7 & 96.6 & 83.8 & 83.8 & 88.1 & 88.1 & 96.7 & 96.6 & $96.6 \mathrm{~g}$ & 96.6 & 11 \\
\hline 12 & 3.4 & 3.9 & 3.4 & 4.0 & 3.8 & 3.1 & 2.1 & 1.2 & 0.9 & 1.3 & 2.2 & & | 98.7 & 98.2 & 97.8 & 97.2 & 97.7 & 97.5 & 82.9 & 82.9 & 87.4 & 87.4 & 97.7 & 97.5 & $97.5 \mathrm{~g}$ & 97.5 & 12 \\
\hline 13 & 4.5 & 5.0 & 4.5 & 5.1 & 4.9 & 4.3 & 3.2 & 1.8 & 1.5 & 1.7 & 3.0 & 1.3 & & 97.7 & 97.2 & 96.6 & 97.1 & 96.9 & 82.4 & 82.4 & 86.6 & 86.6 & 97.1 & 96.9 & $96.9 \mathrm{~g}$ & 96.9 & 13 \\
\hline 14 & 4.6 & 4.8 & 4.6 & 4.9 & 5.0 & 4.4 & 3.5 & 2.3 & 2.0 & 2.4 & 3.3 & 1.8 & 2.4 & & 96.8 & 96.7 & 97.2 & 97.1 & 82.7 & 82.7 & 86.9 & 86.9 & 97.2 & 97.1 & 97.19 & 97.1 & 14 \\
\hline 15 & 4.8 & 5.0 & 4.5 & 5.5 & 4.9 & 4.8 & 3.5 & 2.5 & 2.3 & 2.6 & 3.3 & 2.3 & 2.9 & 3.3 & & 96.4 & 97.1 & 96.9 & 83.0 & 83.0 & 86.7 & 86.7 & 97.1 & 96.9 & $96.9 \mathrm{~g}$ & 96.9 & 15 \\
\hline 16 & 5.5 & 6.3 & 5.8 & 6.4 & 6.2 & 5.5 & 4.5 & 3.0 & 2.8 & 3.1 & 4.3 & 2.8 & 3.4 & 3.2 & 3.6 & & 96.5 & 96.4 & 81.7 & 81.7 & 85.9 & 85.9 & 96.5 & 96.4 & $96.4 \mathrm{~g}$ & 96.4 & 16 \\
\hline 17 & 4.6 & 5.1 & 4.6 & 5.3 & 5.0 & 4.4 & 3.4 & 2.4 & 2.4 & 2.8 & 3.4 & 2.4 & 3.0 & 2.9 & 3.0 & 3.5 & & 98.7 & 82.7 & 82.7 & 87.4 & 87.4 & 100.0 & 98.7 & $98.7 \mathrm{~g}$ & 98.7 & 17 \\
\hline 18 & 4.5 & 5.0 & 4.5 & 5.4 & 4.9 & 4.5 & 3.5 & 2.5 & 2.5 & 2.9 & 3.5 & 2.5 & 3.1 & 3.0 & 3.1 & 3.6 & 1.3 & & 83.1 & 83.1 & 87.3 & 87.3 & 98.7 & 100.0 & 100.01 & 100.0 & 18 \\
\hline 19 & 19.0 & 19.2 & 18.6 & 19.2 & 18.7 & 18.7 & 19.7 & 19.7 & 19.7 & 19.7 & 18.9 & 20.2 & 20.8 & 20.4 & 20.0 & 21.7 & 20.4 & 19.9 & & 100. & 85.3 & 85.3 & 82.7 & 83.1 & 83.18 & 83.1 & 19 \\
\hline 20 & 19.0 & 19.2 & 18.6 & 19.2 & 18.7 & 18.7 & 19.7 & 19.7 & 19.7 & 19.7 & 18.9 & 20.2 & 20.8 & 20.4 & 20.0 & 21.7 & 20.4 & 19.9 & 0.0 & & 85.3 & 85.3 & 82.7 & 83.1 & 83.18 & 83.1 & 20 \\
\hline 21 & 13.2 & 12.8 & 13.6 & 13.5 & 13.7 & 13.3 & 13.7 & 13.8 & 13.8 & 14.3 & 13.3 & 14.2 & 15.2 & 14.9 & 15.0 & 16.0 & 14.2 & 14.3 & 16.9 & 16.9 & & 100 & 87.4 & 87.3 & 87.38 & 87.3 & 21 \\
\hline 22 & 13.2 & 12.8 & 13.6 & 13.5 & 13.7 & 13.3 & 13.7 & 13.8 & 13.8 & 14.3 & 13.3 & 14.2 & 15.2 & 14.9 & 15.0 & 16.0 & 14.2 & 14.3 & 16.9 & 16.9 & 0.0 & & 87.4 & 87.3 & 87.38 & 87.3 & 22 \\
\hline 23 & 4.6 & 5.1 & 4.6 & 5.3 & 5.0 & 4.4 & 3.4 & 2.4 & 2.4 & 2.8 & 3.4 & 2.4 & 3.0 & 2.9 & 3.0 & 3.5 & 0.0 & 1.3 & 20.4 & 20.4 & 14.2 & 14.2 & & | 98.7 & $98.7 \mathrm{~g}$ & 98.7 & 23 \\
\hline 24 & 4.5 & 5.0 & 4.5 & 5.4 & 4.9 & 4.5 & 3.5 & 2.5 & 2.5 & 2.9 & 3.5 & 2.5 & 3.1 & 3.0 & 3.1 & 3.6 & 1.3 & 0.0 & 19.9 & 19.9 & 14.3 & 14.3 & 1.3 & & 100.01 & 100.0 & 24 \\
\hline 25 & 4.5 & 5.0 & 4.5 & 5.4 & 4.9 & 4.5 & 3.5 & 2.5 & 2.5 & 2.9 & 3.5 & 2.5 & 3.1 & 3.0 & 3.1 & 3.6 & 1.3 & 0.0 & 19.9 & 19.9 & 14.3 & 14.3 & 1.3 & 0.0 & & 100 & 25 \\
\hline 20 & 4.5 & 5.0 & 4.5 & 5.4 & 4.9 & 4.5 & 3.5 & 2.5 & 2.5 & 2.9 & 3.5 & 2.5 & 3.1 & 3.0 & 3.1 & 3.6 & 1.3 & 0.0 & 19.9 & 19.9 & 14.3 & 14.3 & 1.3 & 0.0 & 0.0 & & \\
\hline & 1 & 2 & 3 & 4 & 5 & . & 1 & 8 & 9 & 10 & 11 & 12 & 13 & 14 & 15 & 16 & 17 & 18 & 19 & 20 & 21 & 22 & 23 & 24 & 25 & 26 & \\
\hline
\end{tabular}

JQ906552.1AchickenIEgypt114915V/2011 JQ906556.1AchickenEEgyt1248/2012 KF881640.1 A/chickenEgyptS7022D12013 KJ781215.1 Achicken/Egypt13139V12013 MH182633.1 AlchickenEgypt5/2014 KX000718.1 AcchickenEgyptF9516B/2014 KX000853.1 Achicken/EgyptS10916B/2015 KX000793.1 AlchickenEEgyptD10945E/2015 KX000750.1 A IchickenIEgyptS10939E/2015 KX783417.1 Achicken/srael/214/2016 MH997629.1 AlchickenEEgytA-ch1/2017 INN897765.1 A AchickenEEgyptV69/2017 MN897767.1 AlchickenEEgyptV2479/2017 MK942474.1 Alchicken/srael/303070/2018 MK968882.1 A/chickenEgypt/AB222018 MK212394.1 AchickenEEgyptV152712018 MN897781.1 AlchickenEgyptFAO-S18/2019 MN897782.1 AchickenEEgyptV263012019 HQ871933.1 Alchicken/Korea/GH2/2007 GU053186.1 Achicken/Korea/MS96-CE6/96 KF 188366.1 Alchicken/Hong Kong/G9/97 AF156373.1 A AChicken/Hong Kong/G9/97 MT319757 AChickenEEgytKing-mariot18 MT319758 ACChickenEEgyptMatrouh/19 MT319759 ACChickenEEyptNorth-coast119 MT319760 ACChickenEEgyptBahig/19

Figure 4. Genetic identity between circulating LPAI H9N2 and the selected four isolates (blue text box). 


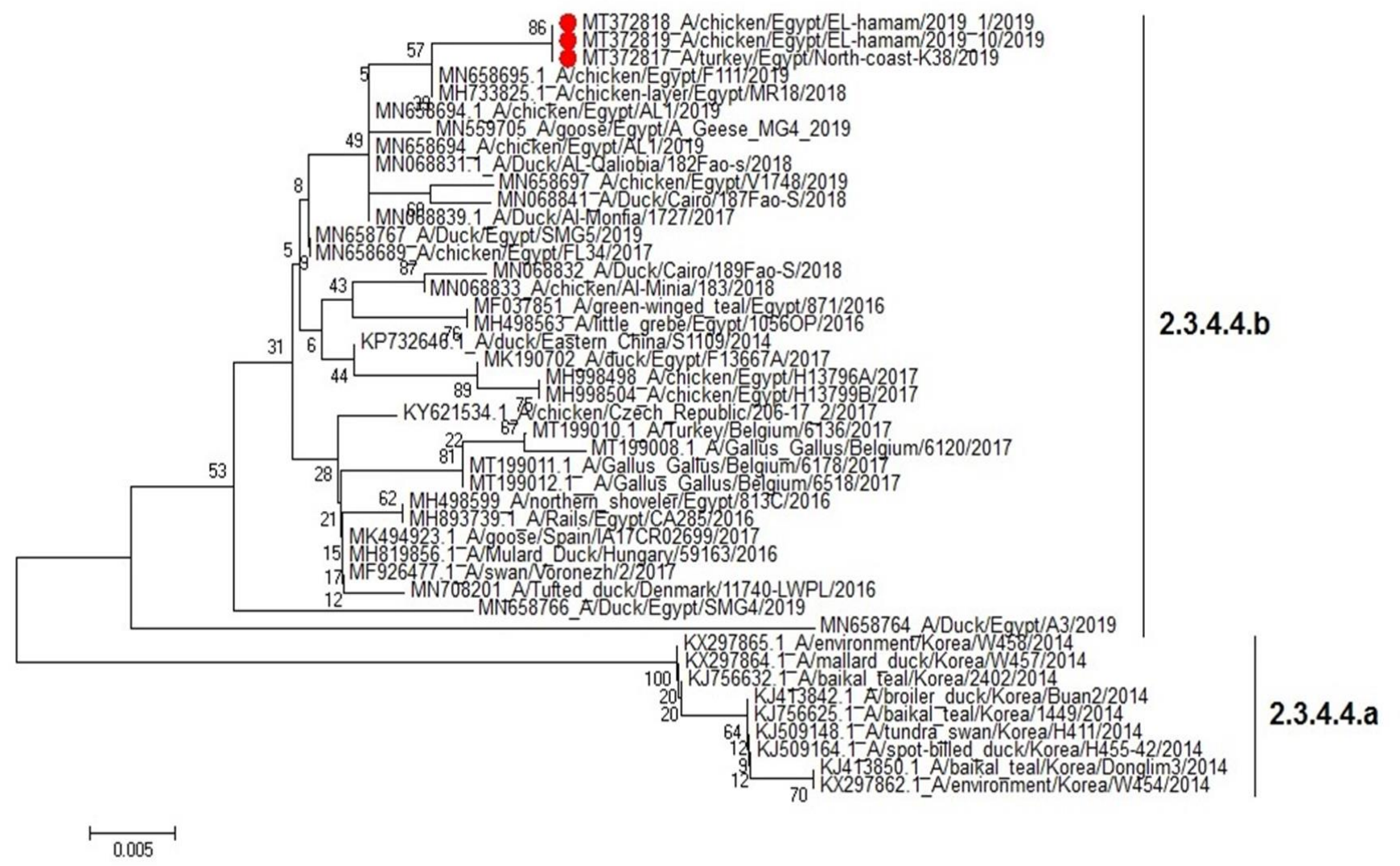

Figure 5. Phylogenetic tree showing the genetic relationships between circulating HPAI H5N8 and the selected three isolates for the HA gene (indicated by red dots).

Percent Identity

\begin{tabular}{|c|c|c|c|c|c|c|c|c|c|c|c|c|c|c|c|c|c|c|c|c|c|c|c|c|c|}
\hline & 1 & 2 & 3 & 4 & 5 & 6 & 7 & 8 & 9 & 10 & 11 & 12 & 13 & 14 & 15 & 16 & 17 & 18 & 19 & 20 & 21 & 22 & 23 & 24 & \\
\hline 1 & & 6 & 99.3 & 98.6 & 98.9 & 7.9 & 97.5 & 9 & 97.9 & 98.6 & 98.9 & 93.3 & 92.9 & 93.3 & 93.6 & 93.6 & 92.9 & 98.2 & 98.2 & 98.2 & 98.2 & 98.2 & 98.6 & 98.6 & 1 \\
\hline 2 & 4 & & 3.9 & 2 & 98.6 & & 97.2 & 5 & 97.5 & & 98.6 & 3.3 & 92.9 & 3 & 93.6 & 93.6 & 92.9 & 97.9 & 97.9 & 97.9 & 97.9 & 9 & 32 & & 2 \\
\hline 3 & & 1 & & & 99.6 & 6 & 98.2 & 98.6 & 98.6 & 99.3 & 99.6 & 94.0 & 93.6 & 94.0 & 3 & 94.3 & 93.6 & \begin{tabular}{|l|}
98.2 \\
\end{tabular} & 98.2 & 98.2 & 98.9 & .9 & 99.3 & & 3 \\
\hline 4 & & 8 & 7 & & 99.6 & 6 & 98.2 & 98.6 & 98.6 & 99.3 & 98.9 & 94.0 & 93.6 & 94.0 & 94.3 & 94.3 & 93.6 & 98.9 & 98.9 & 98.9 & 99.6 & 99.6 & 100.0 & .0 & 4 \\
\hline 5 & & 4 & 0.4 & 0.4 & & 98.9 & 98.6 & 98.9 & 98.9 & 99.6 & 99.3 & 94.3 & 94.0 & 3 & 94.7 & 94.7 & 94.0 & 6 & 98.6 & 98.6 & 99.3 & 3 & 99.6 & & 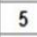 \\
\hline 6 & 2 & 2.5 & 4 & 1.4 & 1.1 & & 7.5 & 97.9 & 98.6 & 98.6 & 98.2 & 93.3 & 92.9 & 93.3 & 93.6 & 93.6 & 92.9 & 97.5 & 97.5 & .5 & 2 & 2 & 6 & & 6 \\
\hline 7 & 2. & 2.9 & 1.8 & 1.8 & 1.4 & 5 & & 99.6 & 98.2 & 98.9 & 97.9 & 93.6 & 93.3 & 93.6 & 94.0 & 94.0 & 93.3 & 97.2 & 97.2 & 97.2 & 97.9 & 97.9 & 98.2 & 2 & 7 \\
\hline 8 & & 5 & 4 & & 1.1 & 2 & 4 & & & 99.3 & 98.2 & 94.0 & 93.6 & 94.0 & 3 & 94.3 & 93.6 & 5 & 97.5 & .5 & 98.2 & 2 & 6 & & 0 \\
\hline 9 & 2.2 & 2.5 & 1.4 & 1. & 1.1 & 1.4 & 8 & 1. & & 99.3 & 98.9 & 94.0 & 93.6 & 94.0 & 94.3 & 94.3 & 93.6 & 97.5 & 97.5 & 97.5 & 98.2 & 98.2 & 98.6 & 6 & 9 \\
\hline 10 & 1. & 8 & 0.7 & 0 & 0.4 & 4 & 1.1 & 0 & & & 98.9 & 94.7 & 94.3 & 94.7 & 95.0 & 95.0 & 94.3 & 98.2 & 98.2 & 98.2 & 98.9 & 98.9 & 99.3 & 99.3 & 10 \\
\hline 11 & & 1.4 & 0.4 & 11 & 0.7 & 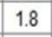 & 2 & & & 1.1 & & 1038 & 93.3 & 93.6 & 94.0 & 94.0 & 93.3 & 97.9 & 97.9 & 97.9 & 98.6 & 98.6 & .9 & & \\
\hline 12 & 7.2 & 7.2 & 6.4 & 6.4 & 6.0 & 7.1 & 6.8 & 64 & 6. & 5.6 & 6.8 & & 99.6 & 100.0 & 99.6 & 99.6 & 99.6 & 92.9 & 92.9 & 92.9 & 93.6 & 93.6 & 94.0 & 94.0 & 12 \\
\hline 13 & 7. & 7.6 & 6. & 6.8 & 6.4 & & & & 6. & & & 4 & & 99.6 & 99.3 & 99.3 & 100.0 & 92.6 & 92.6 & 6 & 3 & 93.3 & 6 & & 15 \\
\hline 14 & 7. & 7.2 & 6.4 & 6.4 & 6. & 7.1 & 6. & 6.4 & 6. & 5 & 6. & 0.0 & 4 & & 99.6 & 99.6 & 99.6 & 92.9 & 92.9 & 92.9 & 93.6 & 93.6 & 94.0 & 9 & 4 \\
\hline 15 & 6. & 6.8 & 6.0 & 6.0 & 5.6 & 6.8 & 6.4 & 60 & 6. & 5.2 & 6.4 & 0.4 & 0.7 & 0.4 & & 100.0 & 99.3 & 93.3 & 93.3 & 93.3 & 94.0 & 94.0 & 94.3 & 94.3 & 15 \\
\hline 16 & 6. & 6.8 & 6.0 & 6.0 & 5.6 & & 8 & & 6. & & & & & & & & 99.3 & 93.3 & 93.3 & 93.3 & 94.0 & 94.0 & 94.3 & & 16 \\
\hline 17 & 7. & 7.6 & 6.8 & 68 & 6.4 & 7.5 & 7.2 & 6.8 & 6 & 6. & 7.2 & 0 & 0.0 & 0.4 & 0.7 & 0.7 & & 92.6 & 92.6 & 92.6 & 93.3 & 93.3 & 93.6 & 93.6 & 17 \\
\hline 18 & 1. & 2.2 & 1.8 & 1.1 & 1.4 & 2.5 & 2.9 & 2.5 & 2. & 1.8 & 2. & 7.6 & 8.0 & 7.6 & 2 & 7.2 & 3.0 & & 100.0 & 100.0 & 99.3 & 99.3 & 98.9 & 98.9 & 18 \\
\hline 19 & 1. & $2 ?$ & 1.8 & 1.1 & 1.4 & & 2 & & & 1.8 & & & & & & & & 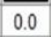 & & 100.0 & 99.3 & 99.3 & 98.9 & 98.9 & 19 \\
\hline 20 & 1.8 & 2.2 & 1.8 & 1.1 & 1.4 & 2.5 & 2.9 & 2.5 & 2.5 & 1.8 & 2.2 & 7.6 & 8.0 & 7.6 & 7.2 & 7.2 & 8.0 & 0.0 & 0.0 & & 99.3 & 99.3 & 98.9 & 98.9 & 20 \\
\hline 21 & 1.8 & 2.2 & 1.1 & 0.4 & 0.7 & 1.8 & 2.2 & 1.8 & 1.8 & 1.1 & 1.4 & 6.8 & 7.2 & 6.8 & 4 & 6.4 & 7.2 & \begin{tabular}{|l|}
0.7 \\
\end{tabular} & 0.7 & 0.7 & & 100.0 & 99.6 & 99.6 & 21 \\
\hline 22 & 1.8 & 2.2 & 1.1 & 0.4 & 0.7 & 1.8 & 2.2 & 1.8 & 1.8 & 1.1 & 1.4 & 68 & 7.2 & 6.8 & 6.4 & 6.4 & 7.2 & 0.7 & 0.7 & 0.7 & 0.0 & & 99.6 & 99.6 & 22 \\
\hline 23 & 1.4 & 1.8 & 0.7 & 0.0 & 0.4 & 1.4 & 1.8 & 1.4 & 1.4 & 0.7 & 1.1 & 6.4 & 6.8 & 6.4 & 6.0 & 6.0 & 6.8 & 1.1 & 1.1 & 1.1 & 0.4 & 0.4 & & 100.0 & 23 \\
\hline \multirow[t]{2}{*}{24} & 1.4 & 1.8 & 0.7 & 0.0 & 0.4 & 1.4 & 1.8 & 1.4 & 1.4 & 0.7 & 1.1 & 6.4 & 6.8 & 6.4 & 6.0 & 6.0 & 6.8 & 1.1 & 1.1 & 1.1 & 0.4 & .4 & 0.0 & & 24 \\
\hline & 1 & 2 & 3 & 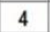 & 5 & 6 & 7 & 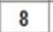 & 8 & 10 & II & 12 & 13 & 14 & 15 & 16 & 17 & 18 & 19 & 20 & 21 & 22 & 23 & 24 & \\
\hline
\end{tabular}

MT199012A/Gallus Gallus/Belgium/6518/17 MT199010 A/Turkey/Belgium/6136/17 MK494923 Avgoose/Spain/A17CR02699/17 MN658694 Avchicken/Egypt/AL1/19 MN658767 ADDuckEgyptSMG5/19 MN068832ANDuckJCairo/189Fa0-S/18 MH998498 Avchicken/EgyptH13796A17 MK190702 AdduckEEyptF13667A117 MF037851A/green-winged teal/Egy/871/16 KP732646 AdduckJEastern China/S1109/14 MH893739 ARRails/EgyptCA285/16 KJ413842 Avbroiler duck/Korea/Buan2/14 KJ413850A/baikal teal/Korea/Donglim3/14 KJ756625 Abaikal teal/Korea/1449/14 KX297865 AvenvironmentKorea/W458/14 KX297864 A/mallard duck/Korea/W457/14 KX297862 AvenvironmentKorea/W454/14 MT372817Aturkey/Egy/North-coast-K38/19 MT372818A/chicken/Egy/EL-hamam/19-1 MT372819A/chicken/Egy/EL-hamam/19-10 MN658695 A/chicken/EgyptF111/19 MH733825Avchicken-layer/EgyptMR18/18 MN068839 ANDuckJAI-Monfia/1727/17 MN658694 Alchicken/Egypt/AL1/19

Figure 6. Genetic identity between circulating HPAI H5N8 and the selected three isolates (blue text box). 


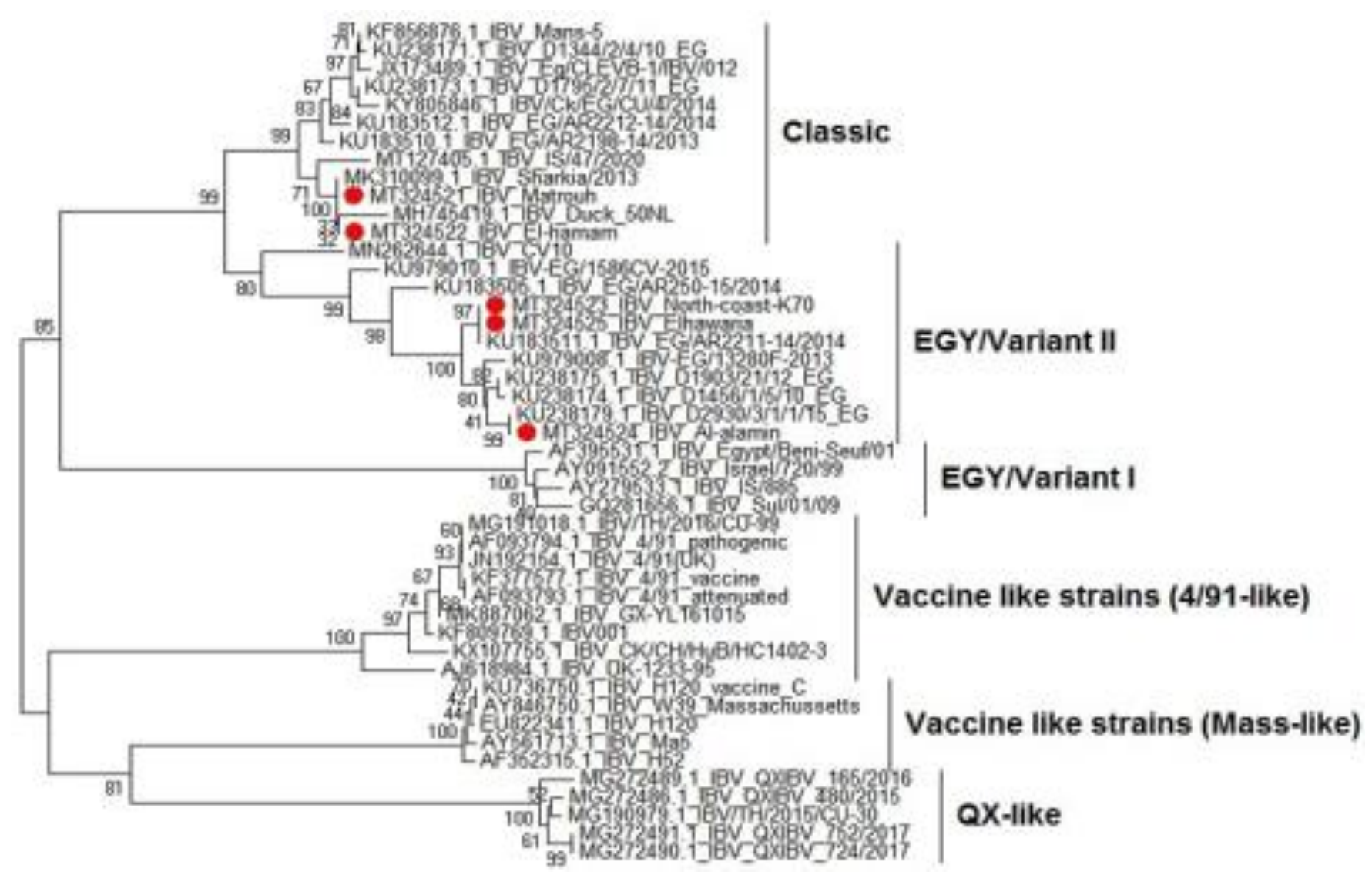

Figure 7. Phylogenetic tree showing the genetic relationships between representative IB viruses and the selected five isolates for the S1 gene (indicated by red dots) IB: Infectious Bronchitis.

\begin{tabular}{|c|c|c|c|c|c|c|c|c|c|c|c|c|c|c|c|c|c|c|c|c|c|c|c|c|c|c|c|c|c|c|c|}
\hline \multicolumn{32}{|c|}{ Percontionety } \\
\hline & & $i$ & $\overline{2}$ & 3 & 4 & 5 & $\theta$ & 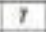 & 7 & 9 & 10 & 11 & 12 & 13 & $M$ & 15 & 16 & $\pi$ & 18 & 10 & 20 & 21 & 22 & D & 24 & 28 & 26 & 27 & 2月 & & \multirow{6}{*}{ 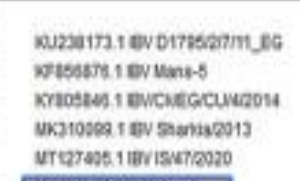 } \\
\hline & 1 & & 20.9 & פ3 & 18.6 & 05.8 & DS6 & 96.6 & 79.7 & 75.5 & 748 & 012 & 804 & 917 & BS & AS: & as: & 105: & ats & 740 & 720 & 749 & 750 & 750 & 787 & 750 & 790 & 710 & 71.8 & 1 & \\
\hline & 2 & 0.5 & & anili & 10.6 & 851 & 186 & 96.6 & 792 & 750 & 70.5 & 02 & 804 & 917 & 105 & 158 & 018 & 155 & ats & 742 & 763 & 742 & 745 & 750 & 797 & 730 & 750 & 703 & 71.3 & 2 & \\
\hline & ( & 07 & 13 & & 18.8 & 349 & Ba & 850 & 790 & 740 & 745 & ats & 187 & 314 & 80 & 185.0 & BSB & $\mu_{0}$ & 05.9 & T43 & 740 & 743 & 745 & 743 & 780 & 763 & 743 & 713 & 71.3 & 3 & \\
\hline & 4 & 28 & 35 & 43 & & 37.5 & 1000 & no0e & 700 & 797 & 725 & 192 & 804 & 912 & 100 & 45.) & 030 & 150 & ato & 795 & 785 & 78.9 & 797 & 750 & 792 & 76.5 & 749 & 72.1 & 70.0 & 4 & \\
\hline & 3 & 48 & is & 0.4 & 25 & & 975 & v.5. & 770 & 787 & 765 & 677 & 800 & 999 & Mo & 15.) & 000 & 153 & Oeno & 793 & $72 \pi$ & 75.7 & 760 & 760 & 732 & 700 & 750 & $n 3$ & 721 & 5 & \\
\hline & 9 & 29 & 29 & $\omega$ & 00 & 25 & & 000 & 780 & 787 & 75.5 & 032 & 804 & 912 & 100 & 15. & $\cos 0$ & 150 & $a s 0$ & 785 & 785 & 79.9 & $78 \pi$ & 750 & 782 & 705 & 74.5 & R1 & 718 & a & 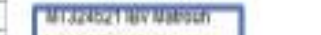 \\
\hline & 7 & $2 s$ & 75 & a) & 00 & 25 & 00 & & 780 & 787 & 758 & ต2 & 100.4 & 912 & mo & 45.2 & 00 & 160 & ano & T35: & 75.5 & 75.5 & 787 & 78.0 & 792 & 70.5 & 76.5 & 721 & 71.8 & 7 & 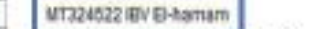 \\
\hline & 1 & $201:$ & 2999 & 33 & $28 \pi$ & 273 & $2 \pi$ & 287 & & 90.5 & 300 & 730 & 750 & 795 & 74) & 735 & 743 & 725 & 76.3 & 6.4 & 60.4 & 69.4 & eat & 711. & 723 & 725 & (72) & 160 & 606 & 1 & 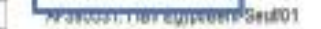 \\
\hline & 9 & 20 & $20.3:$ & 207. & 281 & 27.8 & 291 & 291 & 15 & & 385 & 73 & 74 & 732 & 790 & 74) & 750 & 74) & 750 & 698 & 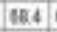 & 624 & $\cos 1$ & 700. & 721 & 727 & 721 & 09 & 1098 & 4 & 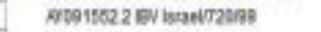 \\
\hline & 10 & 207 & 210 & 311 & 296 & 28.1 & 236 & 296 & 20 & 18 & & 725 & 740 & 750 & 743 & 735 & 743 & 725 & 74.7 & 6.6. & 60.4 & 6.0 & $\cos 4$ & 70.3 & 716 & 710 & 71.6 & 64 & 1098 & 10 & А27963.1 EV KAES \\
\hline & t1 & 130. & 120. & 128 & 129 & 125 & 129 & 127 & 324 & 280 & 342 & & B6. & 819 & 803 & 35.1 & 953 & Reg & 953 & 723 & 72) & 721 & 721 & 702 & 745 & 208 & 720 & $\pi 28$ & 721 & 11 & 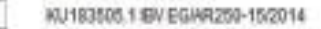 \\
\hline & 12 & 103 & 103 & 112 & 103. & 109 & 10.3 & 10.3 & $3 m 2$ & 206 & 318 & 41 & & 028 & 100 & 981 & 839 & ReA & 929 & 730 & $n 0$ & 730 & $\pi 3$ & 762 & 748 & 700 & 738 & $72 B$ & 721 & 12 & 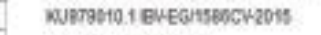 \\
\hline & 13 & 89 & 88 & 0.2 & 906 & 98 & BS & 83 & 29.4 & 297 & 30.2 & BC. & 73 & & 692. & 985 & B92 & 690 & 892. & 707 & 706 & 70.7 & 70.5 & 740 & 700 & 745 & 745 & 721 & 71.4 & 13 & MNESES4.1IEVCFO \\
\hline & 14 & 101 & 15.1 & n.1. & 167 & 18.7. & 107 & 15.7 & 313 & 301 & 31.3 & 49 & 18 & 11.8 & & 98.8 & 1000 & 97.6 & 1000 & 718 & 718 & 718 & 706 & 76.2 & 745 & 73.8 & 730. & 738 & 730 & 14 & 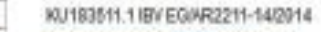 \\
\hline & 15 & $16+1$ & 16.1 & 170 & 107 & 16.7 & 167 & 167 & 320 & 313 & 32.5 & 89 & 73 & 127 & 13 & & 193.8 & 968 & 388 & 71.1 & 71.1 & 71.1 & 70.8 & 748 & 740 & 73.3 & 730 & 730 & 723 & 15 & 1 BN D190BEI/12 EO \\
\hline & 16 & 181 & 15.1 & 15.1 & $15 \mathrm{~T}$ & 15.7 & 167 & 167 & 313 & 301 & 313 & 49 & 18 & 118 & 00 & 13 & & 1075 & 1000 & 718 & $7+8$ & 718 & 716 & 762 & 745 & 738 & 73.5 & $73 B$ & 730 & 16 & 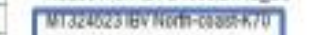 \\
\hline & 17 & 164 & 16.4 & 173 & 17.0 & 167 & 170 & 170 & 325 & 31.3 & 32.8 & 5.4 & 81 & 12.1 & 25 & 12 & 25 & & 97.5 & 708 & 70.8 & 70.8 & 706 & 745 & 74.3 & 735 & 73.3 & 730 & 723 & 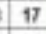 & N184 \\
\hline & 18 & 161 & 15.1 & this & 167 & 15,7 & 157 & 157 & 313 & 301 & 31.3 & 49 & 63 & 11.8 & 0.0 & 13 & 0.0 & 25 & & 718 & 71.8 & 718 & 716 & 75.2 & 745. & 73.8 & 738 & 738 & 73.0 & 18 & บT234525, BVenswar \\
\hline & 19 & 2.4 : & 29.2 & 292 & 27.3 & 270 & 27.3. & 27.3 & 350 & 37.7. & 37.3 & 323 & H.1. & 25.7 & 832 & 34.4 & 332 & 348 & 332 & & 190.5 & 99.5 & 698 & 713 & 725 & 721 & 72.1 & 780 & 75.5 & 19 & EUK22341 1 EVHA120 \\
\hline & 20 & 2841 & 292 & 24.5 & 274 & 270 & 274 & 27.4 & 360 & 37.7 & 37.7 & 324 & 31.2 & 26.1 & 332 & 34.5 & 332 & 349 & 332 & 0.5 & & 199.0 & 903 & 71.3 & 725 & 721 & 72.1 & 745 & 75.0 & 20 & AF352 \\
\hline & 21 & 284. & $20.2:$ & 292 & 27.4 & 27.0 & 27.4 & 27.4 & 360 & 37.7 & 373 & 328 & 31.2 & 25.7 & 332 & 345 & 332 & 349 & 332 & 0.5 & 10 & & 99.3 & 71.1 & 723 & 718 & 718 & 780 & 75.5 & 21 & N5617 \\
\hline & $n$ & $280:$ & 288 & 28.8 & 27.0 & 25.5 & 270 & 27.0 & 36.4 & 881 & 37.7 & 828 & 307 & 260 & 33.6 & 34.9 & 33.5 & 363 & 336 & 0.2 & 08 & 0.8 & & 71.1 & 723 & 71.6 & 71.8 & 748 & 75.2 & $n$ & लвes? \\
\hline & $z$ & 207 & $29.7:$ & 309 & 297 & 30.1 & 297 & 29.7 & 350 & 364 & 362 & 29.6 & 20.6 & 313 & 205 & 30.4 & 22.6 & 30.7 & 29.6 & 348 & 348 & 353 & 362 & & 94.1. & 946 & 94.4 & 7560 & 745 & 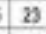 & N619: \\
\hline & 24 & 265 & 28.6 & 297 & 20.4 & 29.4 & 204 & 29.4 & 330 & 83.4 & 34.2 & 316 & 30.3 & 298 & 30.8 & 31.4 & 30.6 & 3.0 & 306 & 328 & 328 & 332 & 33.2 & 62 & & 59.3 & 9800 & 750 & 75.0 & 4 & MKB87ve2. 1 EV OX \\
\hline & 25 & 207 & 29.7 : & 309 & 306 & 302 & 30.6 & 30.5 & 328 & 830 & 338 & 319 & 31.5 & 306 & 319 & 327 & 319 & 322 & 31.9 & 33.6 & 33.5 & 340 & 340 & 56 & 0.7 & & 1948 & 745 & 74.5 & 25 & NA192154.1 EV \&/1(UK) \\
\hline & 26 & $227:$ & 29.7 & 329 & 306 & 201 & 30.6 & 30.6 & 32.9 & 303 & 34.2 & 322 & 31.9 & 30.6 & 322 & 330 & 322 & 326 & 322 & 336 & 337 & 34.1 & 340 & 59 & t.0 & 02 & & 8 & 748 & t) & NF377577.1 G/ 491 vasche \\
\hline & 27 & {$[346]$. 30} & 1554 : & 354 & 342 & $\mid 32.3$ & 342 & 342 & 373 & 37.7 & 37.7 & 33.1 & 330 & 342 & 315 & 327 & 31.5 & 326 & 31.5 & 289 & 29.6 & 289 & 203 & |30.5 & 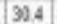 & 312 & 309 & & 30.5 . & 27 & 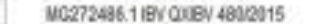 \\
\hline & 28 & 346 & 35.4 & 354 & 347 & 33.1 & 347 & 347 & 373 & 37.9 & 37.3 & 342 & 342 & 350 & 326 & 338 & 326 & 33.8 & 32.6 & 20.1 & 288 & $2 \mathrm{~A} .1$ & 205 & 31.2 & 30.4 & 31.2 & 300 & Et & & & MG272521.118V COUEV 7522A17? \\
\hline & & 1 & $2+2+2+2+3$ & 3 & $"$ & 2 & 0 & 7 & 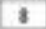 & g & 10 & 11 & 12 & 13 & 14 & 15 & 16 & 17 & 10 & W & $\Delta N$ & 21 & 22 & $\boldsymbol{z}$ & 24 & 25 & 28 & 27 & 28 & & \\
\hline
\end{tabular}

Figure 8. Genetic identity between circulating IB and the selected five isolates (blue text box) IB: Infectious Bronchitis. 


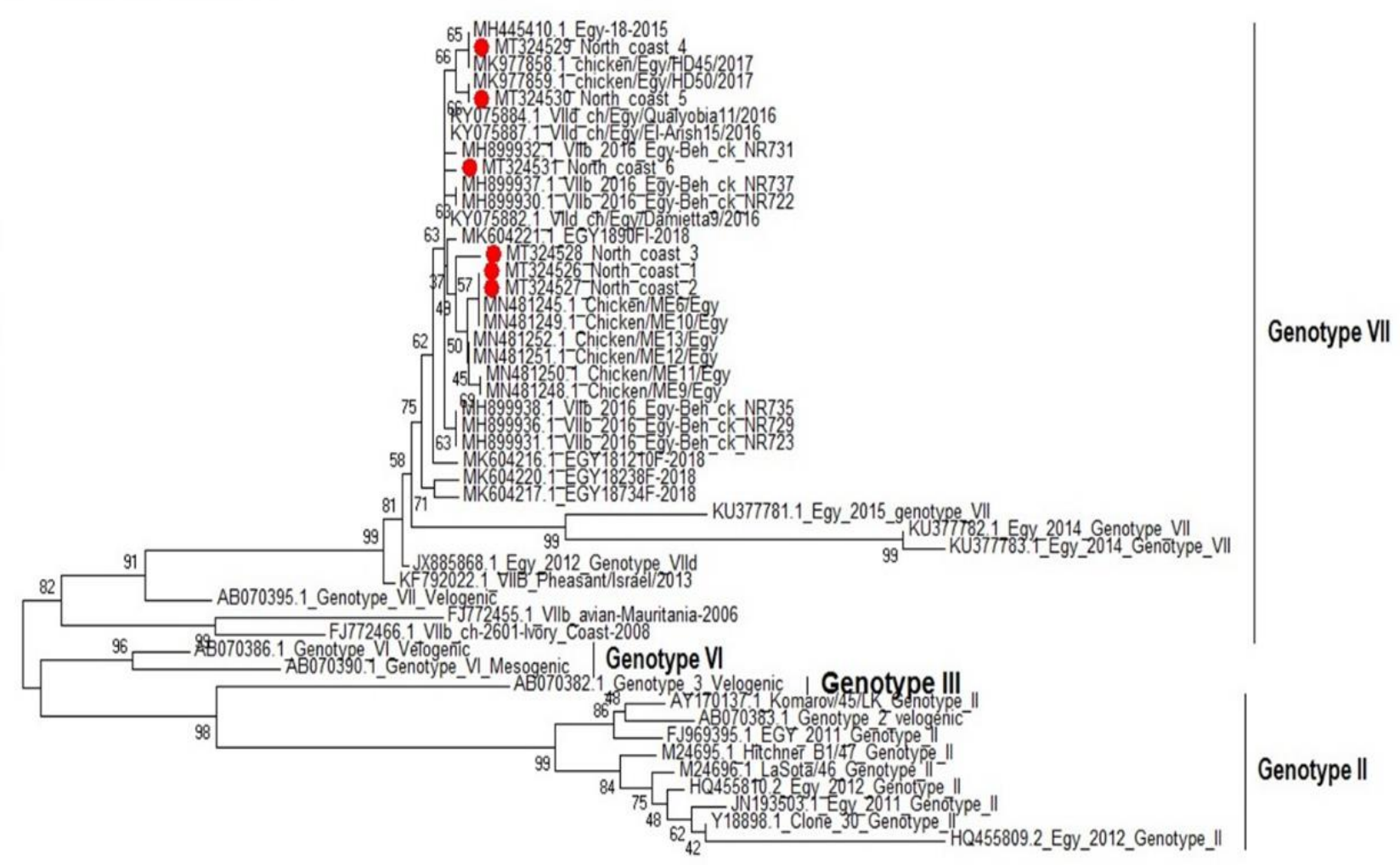

Figure 9. Phylogenetic tree showing the genetic relationships between circulating ND viruses and the selected six isolates for the F gene (indicated by red dots) ND: Newcastle disease.

Percomilantity

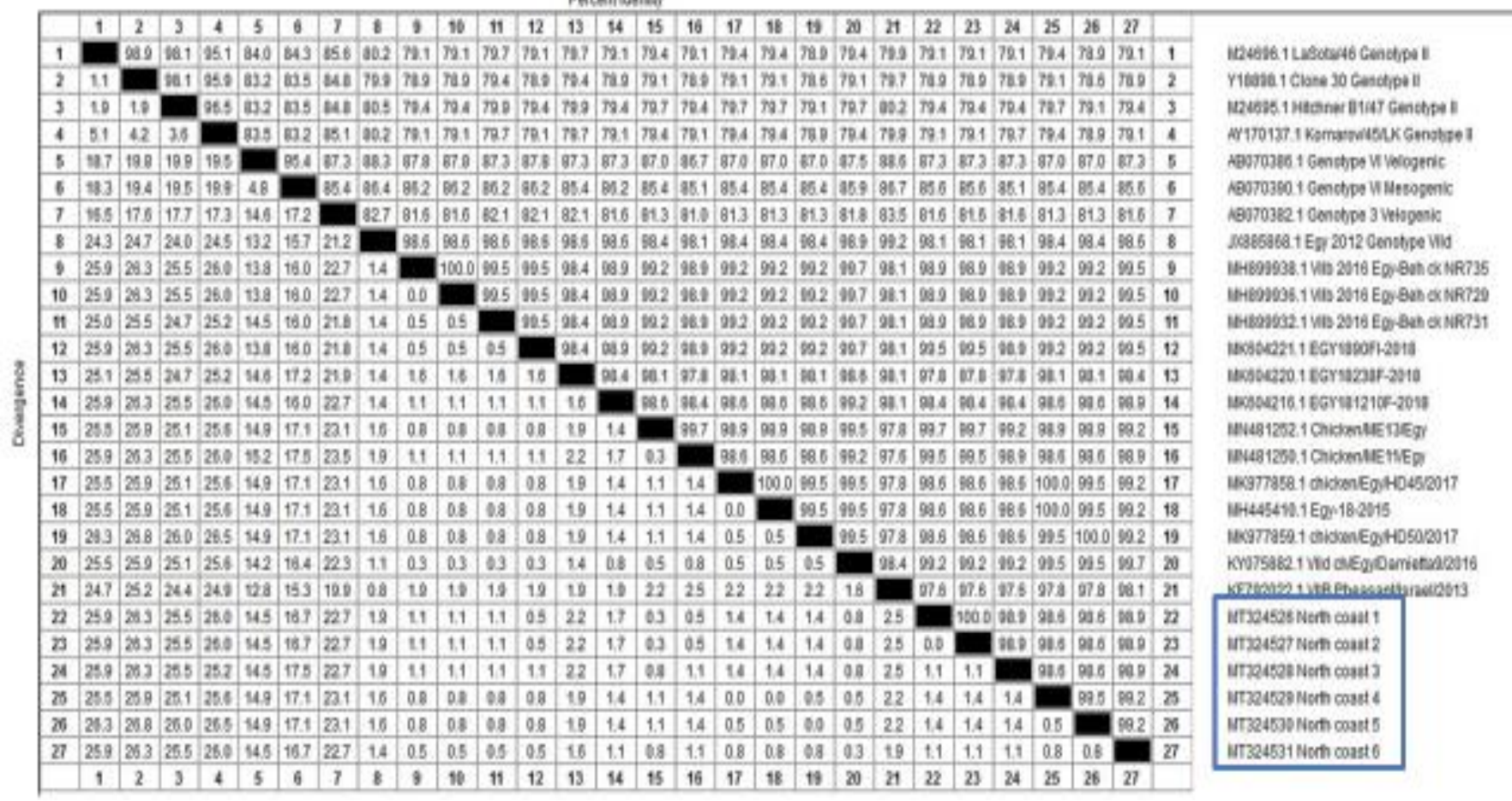

Figure 10. Genetic identity between circulating ND viruses and the selected six isolates (blue text box). ND: Newcastle disease. 
Table 3. Results of RRT-PCR of surveyed poultry flocks.

\begin{tabular}{|c|c|c|c|c|}
\hline \multirow{2}{*}{ virus } & & \multicolumn{2}{|c|}{ AI H9N2 } & \multirow{2}{*}{ Total } \\
\hline & & +ve & -ve & \\
\hline \multirow{2}{*}{ IB } & $+v e$ & 10 & 22 & 32 \\
\hline & -ve & 5 & 6 & 11 \\
\hline \multirow{2}{*}{ ND } & $+v e$ & 4 & 20 & 24 \\
\hline & -ve & 3 & 4 & 7 \\
\hline \multirow{2}{*}{ AI H5N8 } & $+\mathrm{ve}$ & 0 & 9 & 9 \\
\hline & -ve & 0 & 6 & 6 \\
\hline Total & & 22 & 67 & 89 \\
\hline
\end{tabular}

ND: Newcastle disease IB: Infectious bronchitis +ve: Positive -ve: Negative AI H9N2: Avian influenza H9 subtype AI H5N8: Avian influenza H5N8 subtype.

Table 4. Prevalence of different respiratory viruses in poultry flocks in the north coast of Egypt.

\begin{tabular}{lcccc}
\hline Virus & AI H9N2 & IB & ND & AI H5N8 \\
\hline Total flocks & 89 & 43 & 31 & 15 \\
+ ve & 22 & 32 & 24 & 9 \\
percent & $24.7 \%$ & $74.4 \%$ & $77.4 \%$ & $60 \%$
\end{tabular}

ND: Newcastle disease IB: Infectious bronchitis +ve: Positive -ve: Negative AI H9N2: Avian influenza H9N2 subtype AI H5N8: Avian influenza H5N8 subtype.

Table 5. Single and mixed infection with respiratory viruses in the surveyed poultry flocks.

\begin{tabular}{|c|c|c|c|c|c|}
\hline Viruses & AI H9N2 & IB & ND & AI H5N8 & Total \\
\hline Single infection & 8 & 22 & 20 & 9 & 59 \\
\hline Mixed infection with AI H9N2 & - & 10 & 4 & - & 14 \\
\hline
\end{tabular}

ND: Newcastle disease IB: Infectious bronchitis AI H9N2: Avian influenza H9 subtype AI H5N8: Avian influenza H5N8 subtype.

Table 6. Data and GenBank accession numbers of selected virus isolates for sequencing.

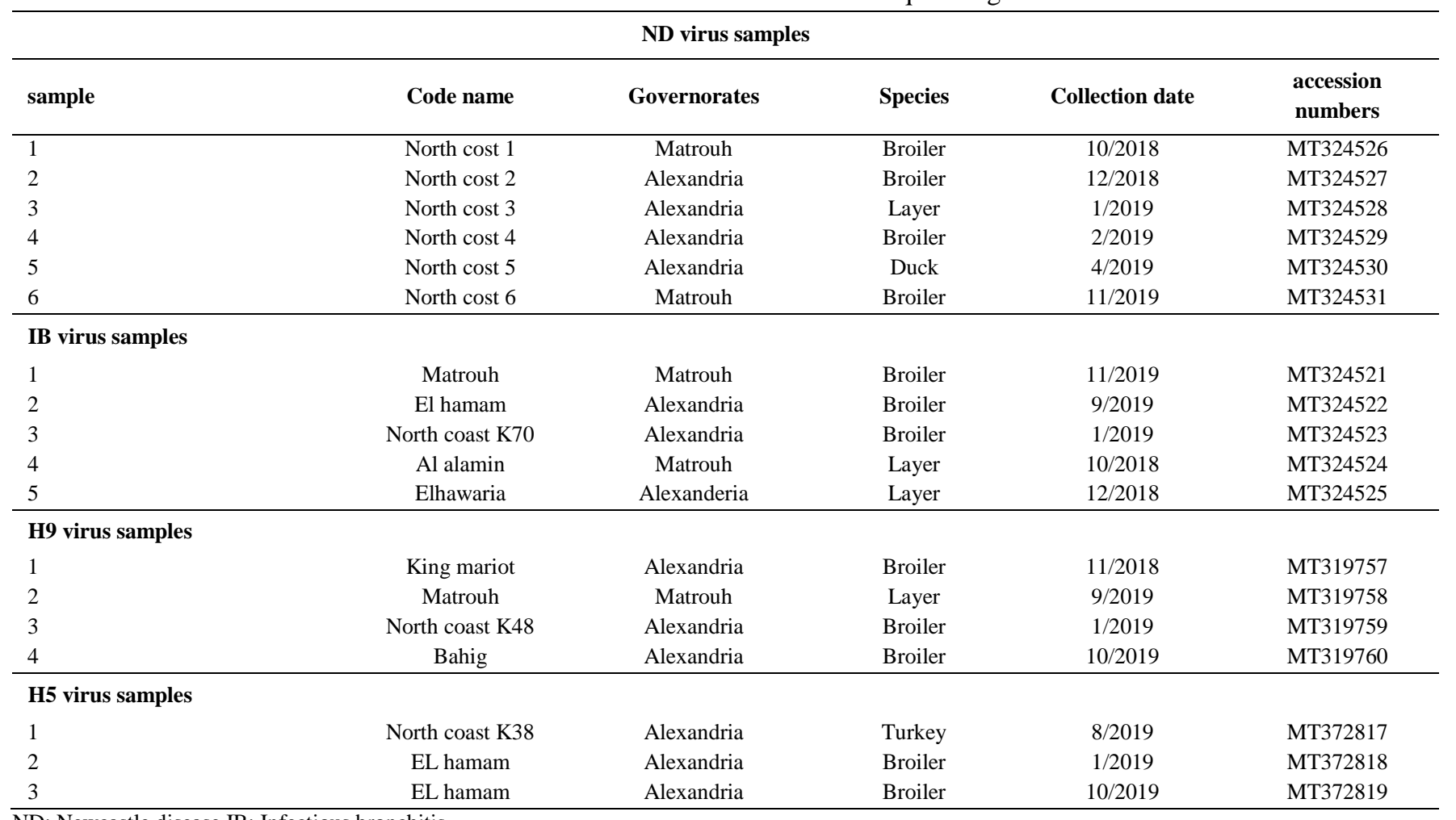




\section{DISCUSSION}

Avian viral respiratory pathogens investigated in the north coast poultry farms were detected in the current study using RRT-PCR. The obtained results indicated that ND viruses had the highest rate of $77.4 \%$ followed by $74.4 \%$, $60 \%$, and $24.7 \%$ for $\mathrm{IB}, \mathrm{H} 5 \mathrm{~N} 8$, and $\mathrm{H} 9 \mathrm{~N} 2$ viruses, respectively. These results matched with those reported by Hassan et al. (2019) who found that 35, 27, 12, 9, and 9 samples out of 39 flocks were positive for AI H5N8, AI H9N2, IB, AI H5N1, and ND viruses, respectively, and detected an increase in the rate of positive flocks for AI H5N8 from $23 \%$ in 2017 to $66.6 \%$ during 2018. Shakal (2013) recorded that IB viruses had the highest incidence rate with $64 \%$ of the total investigated poultry farms, during January-July 2012 at 19 Egyptian governorates. Taher et al. (2017) showed that the incidence of IB, ND, AI H5, and AI H9 was $13.3 \%, 5.6 \%, 2.8 \%$, and $1.1 \%$ respectively. Amer et al. (2018) found that $\mathrm{H} 5, \mathrm{H} 9$, and $\mathrm{H} 5+\mathrm{H} 9$ subtypes were rated as $8.3 \%, 16.7 \%$, and $6.5 \%$ respectively.

Co-infection with circulating H9N2 occurred in 14 poultry flocks, 10 flocks with IB, and 4 flocks with ND viruses. Abd El-Hamid et al. (2018) found that 12 out of 36 H9N2 affected poultry flocks co-infected with H5N1 (19.4\%), ND (11.1\%), and IB viruses (8.3\%). Hassan et al. (2016) showed that mixed infections of IB with AI H9N2 viruses were the most common infection (41.7\%). Amer et al. (2018) detected the mixed infections in chicken flocks of Egypt with AI H9N2 and ND genotype VII viruses. Davidson et al. (2014) and Hassan et al. (2016) reported that natural co-infections of AI H9N2 with ND viruses have occurred in poultry in Egypt.

Regarding the results of sequencing and phylogenetic analysis in the current study, AI H9N2 isolated viruses belonged to G1-lineage circulating in the Middle East, and were clustered with the isolated Egyptian H9N2 viruses during 2011-2019 with the similarity rate of 94.8-100\%. Amer et al. (2018) found that H9 sequences belonged to the G1 lineage clustered with Egyptian H9N2 strains during 2015-2016. Abd El-Hamid et al. (2018) reported that the $\mathrm{H} 9$ isolates were clustered with recent Egyptian isolates of G1/97-like lineage of HA gene sequencing, and were similar to A/Quail/Egypt/113413v/2011 with about 92.3\%-97.1\% similarity rate. Monne et al. (2012) reported that all Egyptian AI H9N2 isolates were grouped within group B of G1-like lineage.

AI H5N8 isolated viruses were closely related to clade 2.3.4.4.b circulating in Egypt from 2014 to 2019 with an identity of $98.9-99.3 \%$. Hassan et al. (2019) revealed that $\mathrm{H} 5 \mathrm{~N} 1$ and $\mathrm{H} 5 \mathrm{~N} 8$ were grouped within Clade 2.2.1.2 and Clade 2.3.4.4b, respectively. WHO (2019) reported that the higher the number of $\mathrm{H} 5 \mathrm{~N} 8$ infected poultry farms, the lower the number of human cases in Egypt (2016: 10; 2017: 3; 2018: 0). Also, Grund et al. (2018) reported that Clade 2.3.4.4b H5N8 viruses were known to have grossly reduced zoonotic propensity versus clade 2.2.1.2 H5N1 (Samir et al., 2015; Ghazi et al., 2016).

Two IB isolated viruses were located with classic strain circulating in Egypt with an identity of 71.8-91.2\%, and the other three IB isolates belonged to EGY/Variant 11 with an identity of 70.6-75.2\%. Abou El-Fetouh et al. (2016) revealed that the results of partial sequencing and phylogenetic analysis of $400 \mathrm{bp}$ of $\mathrm{S} 1$ gene for the isolates of IB viruses were separated into two distinct groups, namely variant 2 and classic. Also, Zanaty et al. (2016) isolated $20 \mathrm{IB}$ viruses, four belonging to the classic group and 16 belonging to the variant group (six isolates with Egy /Var-1 and 10 isolates with Egy /Var -2).

Abd El-Moneim et al. (2012) partially analyzed the $\mathrm{S} 1$ gene of IB virus isolates and found that these isolates were closely related to recent Egyptian IB viruses isolated in northern and middle Egypt, and belonged to genotype variant 2. Meir et al. (2004) found that isolates of IB viruses were closely related to the Israeli nephropathogenic isolate (IS/885/00).

Finally, the selected isolated ND viruses belonged to genotype Vll circulating in Egypt from 2012 to 2018 with an identity of $97.6-100 \%$. Amer et al. (2018) found that NDV isolates belonged to the class II genotype VII. Ramzy (2016) mentioned that genetic classification has divided NDV into two classes (I and II); class I was composed of only 1 genotype (class I, genotype I), and class II was divided into 18 genotypes (class II, genotypes I-XVIII). The predominant genotypes and virulent viruses circulating worldwide were genotypes V, VI, and VII. Genotype VII was associated with the most recent outbreaks in Africa, Asia, and the Middle East.

\section{CONCLUSION}

The current study concluded that the most predominant etiology of respiratory diseases in the north coast of Egypt were ND followed by IB, AI H5N8, and AI H9N2 viruses, furthermore ND and IB viruses isolated in this study were not related genetically to their vaccinal strains. Also, AI H5N8 circulating alone in affected flocks while AI H9N2 circulating alone and/or mixed with either IB (most 
common) or ND viruses (less), finally, The north coast of Egypt is a zone of sustainable development, so there is a need to devise a complete strategy to control the isolated respiratory viruses.

\section{DECLARATIONS}

\section{Acknowledgments}

The authors thank the members of Maryout Research Station, Desert Research Center, Ministry of Agriculture, Egypt. With deeply indebted and grateful to Dr. Ahmed Erfan, Researcher, Reference Laboratory for Veterinary Quality Control on Poultry Production, Agriculture Research Center, Ministry of Agriculture, Egypt.

\section{Competing interests}

The authors declare that they do not have any Competing interests.

\section{Authors' contribution}

Dr. Hanan El-Samahy planned the work, article writing, and revision, Dr. Disouky Mourad designed the protocol and helped in sample collection and laboratory analyses. All authors have read and approved the final manuscript.

\section{REFERENCES}

Abd El-Hamid HS, Ellakany HF, Elbestawy AR, and Gado AR (2018). Molecular characterization of H9N2 avian influenza viruses isolated from commercial broiler chickens in Egypt during 20142015. Alexandria Journal of Veterinary Sciences, 56(2): 54-61. DOI: https://www.doi.org/10.5455/ajvs.220473

Abd El-Moneim AS, Afifi MA, and El-Kady MF (2012). Emergence of a novel genotype of avian infectious bronchitis virus in Egypt. Archives of Virology, 157(12): 2453-2457. DOI: https://www.doi.org/10.1007/s00705-012-1445-1

Abou El-Fetouh MS, Mohamed HM, Nahla AG, Mohamed HA, and Abd Elfattah IE (2016). Pathological studies on infectious bronchitis disease in chickens. Zagazig Veterinary Journal, 44(3): 251-262. DOI: https://www.doi.org/10.21608/zvjz.2016.7879

Adel A, Arafa A, Hussein HA, and El-Sanousi A (2016). Antibody response of commercial vaccinated layers against $\mathrm{H} 9 \mathrm{~N} 2$ viruses in Egypt. Global Veterinaria, 16(6): 557-564. DOI: https://www.doi.org/10.5829/idosi.gv.2016.16.06.10463

Altschul SF, Gish W, Miller W, Myers EW, and Lipmanl DJ (1990). Basic local alignment search tool. Journal of Molecular Biology, 215(3): 403-410. DOI: https://www.doi.org/10.1016/S00222836(05)80360-2

Aly MM, Arafa A, and Hassan MK (2008). Epidemiological findings of outbreaks of disease caused by highly pathogenic H5N1 avian influenza virus in poultry in Egypt during 2006. Avian Diseases, 52(2): 269-277. DOI: https://www.doi.org/10.1637/8166-103007Reg.1.

Amer MM, Asmaa MM, Abdel-Alim GA, Awaad MH, and Kutkat MA (2018). Isolation and identification of H9N2 avian influenza and Newcastle disease viruses co-infections in chicken. Egyptian
Journal of Veterinary Sciences, 49(2): 135-146. DOI: https://www.doi.org/10.21608/ejvs.2018.4963.1043

Ben Shabat M, Meir R, Haddas R, Lapin E, Shkoda I, Raibstein I, Perk S, and Davidson I (2010). Development of a real-time TaqMan RTPCR assay for the detection of H9N2 avian influenza viruses. Journal of Virological Methods, 168(1-2): 72-77. DOI: https://www.doi.org/10.1016/j.jviromet.2010.04.019

Cook JK, Jackwood M, and Jones RC (2012). The long view: 40 years of infectious bronchitis research. Avian Pathology, 41(3): 239-250. DOI: https://www.doi.org/10.1080/03079457.2012.680432

Davidson I, Fusaro A, Heidari A, Monne I, and Cattoli G (2014). Molecular evolution of $\mathrm{H} 9 \mathrm{~N} 2$ avian influenza viruses in Israel. $\begin{array}{llll}\text { Virus } & \text { Genes, } & 48(3) \text { : } & 457-463 .\end{array}$ https://www.doi.org/10.1007/s11262-014-1037-0

De Wit J (2000). Detection of infectious bronchitis virus. Avian Pathology, 29(2): 71-93. DOI: https:// www.doi.org/ 10.1080/ 030 79450094108.

Easterday BC, Hinshaw VS, and Halvorson DA (1997). Influenza. In diseases of poultry, $10^{\text {th }}$ ed. Calnek et al., eds., Ames IA: Iowa State University Press, pp. 583-605.

El-Zoghby EF, Arafa AS, Hassan MK, Aly MM, Selim A, Kilany WH, Selim U, Nasef S, Aggor MG, Abdel Wahab EM et al. (2012). Isolation of H9N2 avian influenza virus from bobwhite quail (Colinus virginianus) in Egypt. Archives of Virology, 157(6): 11671172. DOI: https://www.doi.org/10.1007/s00705-012-1269-z.

Gelb JJ, and Jackwood MW (2008). Infectious Bronchitis. In A Laboratory Manual for the Isolation, Identification and Characterization of Avian Pathogens, 5th ed. L. Dufour-Zavala, D.E. Swayne, J.R. Glisson, J.E. Pearson, W.M. Reed, M.W.Jackwood, and P. Woolcock, eds. American Association of Avian Pathologists, Kennett Square, pp. 146-149. Available at: https://www.vet-library.com/pathology/microbiology/virology/p2224-a-laboratory-manual-for-the-isolation-identification-andcharacterization-of-avian-pathogens-5th-edition.html

Ghazi K, Ahmed K, Rabeh ES, Ahmed SK, Asmaa MM, Zhipeng C, and Mohamed AA (2016). Avian influenza A (H5N1) virus in Egypt. Emerging Infectious Diseases journal, 22(3): 379-388. DOI: https://www.doi.org/10.3201/eid2203.150593

Grund C, Hoffmann D, Ulrich R, Naguib M, Schinköthe J, Hoffmann B, Harder T, Saenger S, Zscheppang K, Tönnies M et al. (2018). A novel European H5N8 influenza A virus has increased virulence in ducks but low zoonotic potential. Emergening Microbes and Infections, 7(1): 1-14. DOI: https://www.doi.org/10.1038/s41426018-0130-1

Hassan KE, El-Kady MF, EL-Sawah AA, Luttermann C, Parvin R, Shany S, Beer M, and Harder T (2019). Respiratory disease due to mixed viral infections in poultry flocks in Egypt between 2017 and 2018: Upsurge of highly pathogenic avian influenza virus subtype H5N8 since 2018. Transboundary and Emerging Diseases, pp. 1-16. DOI: https://www.doi.org/10.1111/tbed.13281

Hassan KE, Shany SA, Ali A, Dahshan AH, El-Sawah AA, and El-Kady MF (2016). Prevalence of avian respiratory viruses in broiler flocks in Egypt. Poultry Science, 95(6): 1271-1280. DOI: https://www.doi.org/10.3382/ps/pew068

Hoffmann B, Hoffmann D, Henritzi D, Beer M, and Harder TC (2016). Riems influenza a typing array (RITA): An RT-qPCR-based low density array for subtyping avian and mammalian influenza a viruses. Scientific Reports, 6(1): 1-10. DOI: https://www.doi.org/10.1038/srep27211

Löndt BZ, Nunez N, Banks J, Nili H, Johnson LK, and Alexander DJ (2008). Pathogenesis of highly pathogenic avian influenza A/turkey/Turkey/1/2005 H5N1 in Pekin ducks (Anas platyrhynchos) infected experimentally. Avian Pathology, 37(6): 619-627. DOI: https://www.doi.org/10.1080/03079450802499126 
Mase M, Imai K, Sanada Y, Sanada N, Yuasa N, Imada T, Tsukamoto K, and Yamaguchi S (2002). Phylogenetic analysis of newcastle disease virus genotypes isolated in Japan. Journal of Clinical Microbiology, $\quad 40(10): \quad 3826-3830 . \quad$ DOI: https://www.doi.org/10.1128/JCM.40.10.3826-3830.2002

Meir R, Maharat O, Farnushi Y, and Simanov L (2010). Development of a real-time TaqMan $®$ RT-PCR assay for the detection of infectious bronchitis virus in chickens and comparison of RT-PCR and virus isolation. Journal of Virological Methods, 163(2): 190-194. DOI: https://www.doi.org/10.1016/j.jviromet.2009.09.014

Meir R, Rosenblut E, Perl S, Kass N, Ayali G, Perk S, and Hemsani E (2004). Identification of a novel nephropathogenic infectious bronchitis virus in Israel. Avian Diseases, 48(3): 635-641. DOI: https://www.doi.org/10.1637/7107.

Mohamed MH, Kumar S, Paldurai A, and Samal SK (2011). Sequence analysis of fusion protein gene of Newcastle disease virus isolated from outbreaks in Egypt during 2006. Virology Journal, 8(1): Article number 237. DOI: https://www.doi.org/10.1186/1743$\underline{422 X-8-237}$

Monne I, Hussein HA, Fusaro A, Valastro V, Hamoud MM, Khalefa RA, Dardir SN, Radwan MI, Capua I, and Cattoli G (2012). H9N2 influenza A virus circulates in $\mathrm{H} 5 \mathrm{~N} 1$ endemically infected poultry population in Egypt. Influenza and Other Respiratory Viruses, 7(3): 240-243. DOI: $\quad$ https://www.doi.org/10.1111/j.17502659.2012.00399.x

Naguib MM, El-Kady MF, Lüschow D, Hassan KE, Arafa AA, ElZanaty A, Hassan MK, Hafez HM, Grund C, and Harder TC (2017). New real time and conventional RT-PCRs for updated moleculardiagnosis of infectious bronchitis virus infection (IBV) in chickens in Egypt associated with frequent co-infections with avian influenza and Newcastle disease viruses. Journal of Virological Methods, 245: 19-27. DOI: https://www.doi.org/10.1016/j.viromet.2017.02.018

Office International Epizootics (OIE) (2005). Manual of diagnostic tests and vaccines for terrestrial animals. Part 2 section 2.1. Chapter, 2.1. Available at: https://www.oecd-ilibrary.org/sites/b3852e6ben/index.html?itemId=/content/component/b3852e6b-en

Radwan MM, Darwish SF, El-Sabagh IM, El-Sanousi AA, and Shalaby MA (2013). Isolation and molecular characterization of Newcastle disease virus genotypes II and VIId in Egypt between 2011 and 2012. Virus Genes, 47(2): 311-316. DOI: https://www.doi.org/10.1007/s11262-013-0950-y

Ramzy N (2016). Viral respiratory diseases of chicken in Egypt. Egyptian Journal of Chemistry and Environmental Health, 2(2): 3844. Available at: http://cehea.org/wp-content/uploads/2017/01/5.pdf

Samir R, Eduardo AB, Marwa Mohamed A, Manal F, Katherine R, Abd Hanaa Abu Elsood E, and Amr K (2015). increased number of human cases of influenza virus A (H5N1) infection, Egypt, 201415. Emerging Infectious Diseases Journal, 21(12): 2171-2173. DOI: https://www.doi.org/10.3201/eid2112.150885.
Shakal M (2013). Current situation of poultry respiratory diseases in Egypt. international conference on animal \& dairy sciences July 2324 , embassy suites las vegas, NV, USA. Journal of Veterinary Science and Technology, 4: 4. DOI: http://www.dx.doi.org/10.4172/2157-7579.S1.002

Shankar BP (2008). Common respiratory diseases of poultry. Veterinary World, 1(7): 217-219. Available at: http://www.veterinaryworld.org/2008/July/10.html.

Slomka MJ, Coward VJ, Banks J, Löndt BZ, Brown IH, Voermans J, Koch G, Handberg KJ, Jørgensen PH, Cherbonnel-Pansart M et al. (2007). Identification of sensitive and specific avian influenza polymerase chain reaction methods through blind ring trials organized in the European Union. Avian Diseases, 51(1): 227-234. DOI: https://www.doi.org/10.1637/7674-063006R1.1

Spackman E, Senne DA, Myers TJ, Bulaga LL, Garber LP, Perdue ML, Lohman K, Daum LT, and Suarez DL (2002). Development of a real-time reverse transcriptase PCR assay for type a influenza virus and the avian $\mathrm{H} 5$ and $\mathrm{H} 7$ hemagglutinin subtypes. Journal of Clinical Microbiology, 40(9): 3256-3260. DOI: https://www.doi.org/10.1128/jcm.40.9.3256-3260.2002

Taher MT, Amer MM, Arafa A, and Saad FE (2017). Epidemiology of viral components causing respiratory problems in broilers in six Egyptian Governorates. Journal of Veterinary Medical Research, 24(1): 308-320. DOI: https://www.doi.org/10.21608/jvmr.2017.43266.

Tamura K, Stecher G, Peterson D, Filipski A, and Kumar S (2013). MEGA 6: molecular evolutionary genetics analysis version 6.0. Molecular Biology and Evolution, 30(12): 2725-2729. DOI: https://www.doi.org/10.1093/molbev/mst197.

Thompson JD, Higgins DG, and Gibson TJ (1994). CLUSTAL W: improving the sensitivity of progressive multiple sequence alignment through sequence weighting, position-specific gap penalties and weight matrix choice. Nucleic Acids Research, 22(22): $\quad 4673-4680 . \quad$ DOI: https://www.doi.org/10.1093/nar/22.22.4673.

Wise MG, Suarez DL, Seal BS, Pedersen JC, Senne DA, King DJ, Kapczynski DR, and Erica Spackman E (2004). Development of a real-time reverse-transcription pcr for detection of Newcastle disease virus RNA in clinical samples. Journal of Clinical Microbiology, $\quad 42(1): \quad 329-338 . \quad$ DOI: https://www.doi.org/10.1128/jcm.42.1.329-338.2004.

World Health Organization (WHO) (2019). Cumulative number of confirmed human cases for Avian Influenza A (H5N1) reported to WHO 2003-2019. Source: WHO /GIP, data in HQ as of 12 February 2019. Available at: https://www.who.int/influenza/human_animal_interface/2019_02_1 2_tableH5N1.pdf?Ua=1

Zanaty A, Arafa AS, Hagag N, and El-Kady M (2016). Genotyping and pathotyping of diversified strains of infectious bronchitis viruses circulating in Egypt. World Journal of Virology, 5(3): 125-134. DOI: https://www.doi.org/10.5501/wjv.v5.i3.125 